\title{
Phylogeny of the Milky Way's inner disk and bulge populations: Implications for gas accretion, (the lack of) inside-out thick disk formation, and quenching
}

\author{
Misha Haywood ${ }^{1}$, Paola Di Matteo ${ }^{1}$, Matthew Lehnert ${ }^{2}$, Owain Snaith ${ }^{3}$, \\ Francesca Fragkoudi ${ }^{1,4}$, and Sergey Khoperskov ${ }^{1}$
}

\author{
${ }^{1}$ GEPI, Observatoire de Paris, PSL Research University, CNRS, Sorbonne Paris Cité, 5 place Jules Janssen, 92190 Meudon, France \\ e-mail: misha.haywood@obspm. fr \\ 2 Sorbonne Université, CNRS, Institut d'Astrophysique de Paris, 98 bis bd Arago, 75014 Paris, France \\ 3 School of Physics, Korea Institute for Advanced Study, 85 Hoegiro, Dongdaemun-gu, Seoul 02455, Republic of Korea \\ ${ }^{4}$ Max-Planck-Institut für Astrophysik, Karl-Schwarzschild-Str. 1, 85741 Garching, Germany
}

Received 13 June 2017 / Accepted 23 February 2018

\begin{abstract}
We show that the bulge and the disk of the Milky Way (MW) at $R \lesssim 7 \mathrm{kpc}$ are well described by a unique chemical evolution and a two-phase star formation history (SFH). We argue that the populations within this inner disk, not the entire disk, are the same, and that the outer Lindblad resonance (OLR) of the bar plays a key role in explaining this uniformity. In our model of a two-phase SFH, the metallicity, $[\alpha / \mathrm{Fe}]$ and $[\alpha / \mathrm{H}]$ distributions, and age-metallicity relation are all compatible with the observations of both the inner disk and bulge. The dip at $[\mathrm{Fe} / \mathrm{H}] \sim 0$ dex seen in the metallicity distributions of the bulge and inner disk reflects the quenching episode in the SFH of the inner MW at age $\sim 8 \mathrm{Gyr}$, and the common evolution of the bulge and inner disk stars. Our results for the inner region of the MW, $R \lesssim 7 \mathrm{kpc}$, are consistent with a rapid build-up of a large fraction of its total baryonic mass within a few billion years. We show that at $z \leq 1.5$, when the MW was starting to quench, transitioning between the end of the $\alpha$-enhanced thick disk formation to the start of the thin disk, and yet was still gas rich, the gas accretion rate could not have been significant. The $[\alpha / \mathrm{Fe}]$ abundance ratio before and after this quenching phase would be different, which is not observed. The decrease in the accretion rate and gas fraction at $z \leq 2$ was necessary to stabilize the disk allowing the transition from thick to thin disks, and for beginning the secular phase of the MW's evolution. This possibly permitted a stellar bar to develop which we hypothesize is responsible for quenching the star formation. The present analysis suggests that MW history, and in particular at the transition from the thick to the thin disk - the epoch of the quenching - must have been driven by a decrease of the star formation efficiency. We argue that the decline in the intensity of gas accretion, the formation of the bar, and the quenching of the star formation rate (SFR) at the same epoch may be causally connected thus explaining their temporal coincidence. Assuming that about $20 \%$ of the gas reservoir in which metals are diluted is molecular, we show that our model is well positioned on the Schmidt-Kennicutt relation at all times.
\end{abstract}

Key words. Galaxy: evolution - Galaxy: disk - Galaxy: bulge - Galaxy: abundances

\section{Introduction}

The stellar-mass density distribution of galaxies shows that a majority of stars in the local universe are in galaxies that have a mass similar to that of the Milky Way (MW; e.g., Papovich et al. 2015; Bell et al. 2017). Recent studies have robustly established that these galaxies form half their mass before $z \sim 1$, or about 8 Gyr ago (Muzzin et al. 2013; van Dokkum et al. 2013; Patel et al. 2013; Papovich et al. 2015). The MW closely follows this general behavior (Snaith et al. 2014) and for our Galaxy, the thick disk alone can explain the early (within the first 34 Gyr) steep mass growth observed in Milky Way-type galaxies. As emphasized recently by Bell et al. (2017), galaxies with stellar mass similar to that of the MW come with a wide variety of morphologies in the local universe, and it is not clear which stellar populations are responsible for this mass growth, in particular because both classical bulges and thick disks are liable to form at these epochs.

In the MW, while evidence for a pseudo-bulge - or a bulge being a bar formed from dynamical instabilities in the disk is now overwhelming, it is still not entirely clear how the disk contributed to its formation. Discrepancies in the properties of the bulge and the disk, and in particular in their chemical properties (see, for a variety of points of view, e.g., Johnson et al. 2013; Di Matteo 2016; Haywood et al. 2016b; McWilliam 2016; Bensby et al. 2017), and the known difficulty to fit a single chemical evolution model to both the bulge and disk (e.g., Tsujimoto \& Bekki 2012; Ballero et al. 2007) have hampered investigations. Hence, when estimating the mass growth of the MW, it may not be entirely clear how the bulge should be taken into account. The concept of stellar population has come in various flavors in the recent literature, with "geometrically" (e.g., Martig et al. 2016) or "chemically" (Bovy et al. 2012) defined stellar populations, which, for the bulge particularly, may be confusing.

A practical definition of a stellar population is a group of stars that presents some homogeneity in either their spatial, kinematic, or chemical properties, or age, or several of these properties (see e.g., Haywood et al. 2013 on how these properties are related). We are looking for a definition that goes beyond the mere identification of stars however, to understand the role 
this population has played in the formation and evolution of the Galaxy. Starting from observables, two steps can be seen as necessary to characterize a population according to this definition: (a) find a criterion unambiguously linked to evolution according to which we can identify stars; (b) relate these stars to a known phase of galactic evolution. For the identification of a population to be robust, the criteria used in (a) must be reliable. Some time after the formation of a population however, spatial and kinematic properties become second-order signatures of a fossil record of a population (Freeman \& Bland-Hawthorn 2002), because various dynamical processes (e.g., bar formation, satellite interactions) are able to reshape distributions, blurring the initial spatial and kinematic signatures. In recent years, chemical abundances have become the genetic markers of stellar populations to astronomers (Fuhrmann 1998; Freeman \& Bland-Hawthorn 2002; Reddy et al. 2003, 2006; Haywood 2008; Nissen \& Schuster 2010; Haywood et al. 2013), and much work is actually being done to both acquire large swaths of new spectroscopic information and to relate this information to Galactic evolution (APOGEE I\&II, WEAVE, 4MOST, MOONS), through proposed techniques such as chemical labeling or tagging (Bland-Hawthorn \& Freeman 2004). Chemical tagging aims at identifying the molecular cloud from which a star originates, thereby identifying the most elementary unit of Galactic evolution. We wish on the contrary to relate the chemical identity of a star to more "holistic" information.

We have shown in Snaith et al. $(2014,2015)$ that the alpha abundances of a population and their evolution with time are directly related to its star formation history (SFH), or in-situ mass growth. If each step of the mass growth of our Galaxy can be related to a corresponding stellar population, through the process described in (a) and (b), then our definition of stellar populations is meaningful and will help us to "reconstruct" the processes out of which our MW formed.

Having clarified the general definition we intend to use for a stellar population, it must be said that, in trying to reconstruct the mass growth history of our Galaxy, we are led to question the parenthood and relationships between the groups of stars that we recognize as populations, in a process akin to phylogeny in biology. This is particularly acute in the MW for the thick disk and bulge, and a now large literature illustrates the question: are the thick disk and bulge the same population? (Meléndez et al. 2008; Alves-Brito et al. 2010; Bensby et al. 2010; Ryde et al. 2010, to name just the first papers on the subject). In trying to answer this question in the following pages, we rely on the same definition of the thick disk as the one given in Haywood et al. (2013), where we showed that a chemically based definition for the thick disk (using alpha element abundances) is the most directly linked to its evolution, because of the tight relation that links alpha abundances to ages, as found in that article. Using this definition, the thick disk considered in this work is unambiguous and concerns stars that are essentially confined to $R<10 \mathrm{kpc}$, are old ( $>9 \mathrm{Gyr}$ ), and are distributed in a thicker disk than the thin disk. We refer the reader to our discussion in Haywood et al. (2013) about the ambiguities of the thick and thin disks nomenclature.

Our article builds on these concepts by showing that there is strong evidence that the bulge and the inner disk (which we define as the thick and the thin disk inside $R \lesssim 7 \mathrm{kpc}$ ) are essentially the same population, and that their chemical evolution can be described by a single and simple model.

The outline of our paper is the following. In the following section, we present our chemical evolution model, and the scenario that goes with it, which was set up in a series of recent papers by our group. The model is confronted with the inner disk constraints in Sect. 3, and with the bulge observations in Sect. 4. In Sect. 5 we discuss arguments that have been invoked to claim that the inner disk and the bulge are the same population, while Sect. 6 presents a discussion about the accretion history of the inner MW. Our conclusions are given in the last section.

\section{Scenario and model}

For reasons that are discussed in Sect. 6, we want to test if the inner MW can be represented by a scenario where most gas accretion has occurred early in the evolution of our Galaxy or before significant star formation started. The model that best approximates this situation is the closed-box model (CBM). Our aim in the present study is to understand how far this simple modeling may be a valid representation of the data to $R \sim 6-7 \mathrm{kpc}$; it is therefore applied to the whole inner disk-bulge stellar system. We first describe the context and limits in which we apply this model, followed by the ingredients and characteristics of the model itself.

\subsection{Landmarks in the Milky Way history and a scenario}

Our study is developed in a context framed by a number of different articles: Haywood et al. (2013, 2016a,b), Lehnert et al. (2014), Di Matteo et al. (2014), Snaith et al. (2014, 2015), Hallé et al. (2015), Di Matteo (2016) but also numerous articles from the literature cited below. We now summarize this context.

(1) In Haywood et al. (2013), we showed that the thick disk formed starting from about 13 Gyr ago to about 9 Gyr ago, during the most intense phase of star formation in the MW (Snaith et al. 2015), in a star burst mode (Lehnert et al. 2014), as can be traced back from the age-alpha relation. The chemical homogeneity of this population (Haywood et al. 2013, 2015) at all ages implies a high level of turbulence in the gas that formed this population.

(2) Fitting the observed age-alpha relation of the inner disk with our GCE model (Snaith et al. 2014, 2015) we were able to infer its SFH showing two distinct phases. The thick disk was formed in the first phase (13-9 Gyr), and represents half the stellar mass of the disk. The thin disk is formed in the second phase, from 7 Gyr to the present day. During the early thick disk phase (age $>11$ Gyr), we found that the SFR density was sufficiently high to generate possible outflows (Lehnert et al. 2014) that may have contributed to pollute the outer $(>10 \mathrm{kpc})$ disk (Haywood et al. 2013).

(3) Haywood et al. (2013) proposed that the disk is better defined by the dychotomy of its radial structure (inner/outer disk) than vertically (thin/thick disk). This was confirmed afterwards by in-situ APOGEE data (Hayden et al. 2015). We argued in Hallé et al. (2015) that, at the formation of the bar, which, in galaxies of the mass of the MW, typically occurs at $z \sim 1$ (Sheth et al. 2008; Melvin et al. 2014), the outer Lindblad resonance (OLR) established a barrier that essentially isolated the inner disk from the outer disk (apart from blurring effects). As shown in Di Matteo et al. (2014), only stars that are within the OLR will participate in the bar and boxy/peanut bulge. Figure A.1 of this article illustrates with a simulation from Hallé \& Combes (2013) that stars beyond the OLR will stay in the outer parts and will not participate in the bar. In the MW, the exact position of the OLR is debated: slightly inside the solar orbit (Dehnen 2000), or further away (e.g., Pérez-Villegas et al. 2017, who, after the work of Portail et al. 2017, proposed that it is situated at $10.5 \mathrm{kpc}$ ). It is however expected that the OLR has 
moved outward as a consequence of the slowdown of the bar, hence it may have formed within the solar orbit and moved further out. In Hallé et al. (2015), we argued that it has maintained the inner disk essentially separated from the outer disk, which developed two separated chemical evolutions. Hence, the OLR delineates two regions of the disk that will mix only a limited amount of their stars. The possible effect of the OLR is therefore a fundamental feature of our analysis because it justifies that the bulge and the inner disk must be the same population, not the bulge and the disk as a whole.

(4) We found in Haywood et al. (2016a) that within $R<6-7 \mathrm{kpc}$, a major cessation (or "quenching") of star formation occurred at the transition from the thick to thin disks, between 9 and 7 Gyr ago. This quenching however did not occur because of gas exhaustion, since in this case, stars formed after the quenching would show chemical discontinuity with stars formed before the quenching. This is not observed: Haywood et al. (2013) have shown that there is a chemical continuity between the thick and thin inner disks. In Haywood et al. (2016a), we argued that the formation of the bar may have been responsible for quenching the star formation. We emphasize that it is the quenching of the star formation rate (SFR) which allowed the transition from the thick to the thin disk. In Khoperskov et al. (2018), we showed that the formation of a bar is indeed able to significantly reduce the SFR of a disc galaxy (by $\sim$ a factor 10), and in a relatively short amount of time ( 1-2 Gyr), in a way at least qualitatively compatible with the MW.

(5) The bulge of the MW is mainly a pseudo-bulge. Its kinematic properties can be explained if it is (mainly) a bar grown from dynamical instabilities in the disk, with a negligible or nonexistent classical bulge (Shen et al. 2010; Kunder et al. 2012; Ness et al. 2013a,b; Di Matteo et al. 2014). Having essentially a disk origin, it must be made of stars of the thick- and thin disks that originate from inside the OLR (Di Matteo et al. 2014, 2015; Di Matteo 2016). The CMD of the bulge as observed by the HST shows a very tight turn-off (Clarkson et al. 2008), suggesting a coeval, old population. However, it has been shown in Haywood et al. (2016b) that the SWEEPS field turn-off can only be compatible with the observed metallicity distribution function (MDF) of the bulge if there is a correlation between age and metallicity, implying that essentially all super-solar metallicity stars in the bulge must be younger than about 8 Gyr (see also Bensby et al. 2013, 2017; Schultheis et al. 2017).

The above results have two general implications. The first one is that if the bulge is a pseudo-bulge formed from the disk inside the OLR, the two must show the same chemical characteristics. As already mentioned, while the problem of the identification of the bulge and the thick disk has been claimed and investigated several times, the aim here is to link the inner disk, including both the thin and thick disks, to the bulge. Second, according to result (2) above, the thick disk represents about half the stellar mass in the inner disk, and this implies that large amounts of gas were available for star formation early (age $>10 \mathrm{Gyr}$ ) in the disk. As we argue in Sect. 5.2, this implies a significant difference from the assumptions of the inside-out paradigm, and opens the way to using a CBM, which we now introduce, as a possible first approximation of this situation.

\subsection{The model}

The CBM we use - or more precisely the simple CBM, which is the CBM with no dependence of the SFH on radius - has been described in Snaith et al. (2015) and assumes (1) a constant
IMF, (2) a consistently well mixed ISM, (3) no inflow or outflows of gas and (4) initially pristine gas with no metals, but no instantaneous recycling approximation, meaning we derive the full information from detailed yields and metallicity-dependent stellar lifetimes.

There are three fundamental differences between our model and standard CBMs:

(1) Closed-box models, when accompanied with a SchmidtKennicutt type of star-formation law, lead to very fast exhaustion of gas and unrealistic age distributions for a disk (see for instance Fraternali \& Tomassetti 2012). In Snaith et al. (2014, 2015), we derived the SFH of the MW inner disk by fitting the model to the age- $[\mathrm{Si} / \mathrm{Fe}]$ relation, but without assuming any SchmidtKennicutt law. As noted in Haywood et al. (2015), because we argued that the age- $[\mathrm{Si} / \mathrm{Fe}]$ derived from local stars is valid for the whole inner disk, the deduced SFH is also expected to hold for the whole Galaxy inside the OLR. As mentioned previously, the derived SFH presents two distinct phases of star formation, corresponding to the thick and thin inner disks. Figure 1 shows the various model distributions: the MDF (top plot), the agemetallicity distribution (middle plot), and the SFH (bottom plot). Two models are shown. The thick curves represent the fiducial model obtained in Snaith et al. (2015), while the thin curves represent distributions obtained in the case of a schematic SFH where we have removed the small-scale variations of the best fit model. In the rest of the article, these two models will be referred to as the fiducial and the smoothed models, respectively.

(2) While classically the CBM has been designed for and compared to the solar vicinity, its application is considered here for the entire bulge and the inner disk, with the aim to test if they can be described as a unique system. The condition of the validity of the CBM to describe the solar vicinity is provided in another article (Haywood et al. in prep.). The "closed-box" is considered here as interesting because it approximates a system strongly dominated by gas at early times, while the fact that it is closed/open has no bearing in this case. Section 6 further discusses how the approximation of the chemical evolution provided by the CBM fits in the context of cosmological gas accretion and Schmidt-Kennicutt law.

(3) The MDF of a CBM is known as a bell-shaped distribution, but this is obtained under the approximation that the recycling of gas ejected by stars is instantaneous, and in this case the shape is independent of the SFH (e.g., Pagel 2009). When the recycling of chemical elements from stars is properly modeled, as it has been in Snaith et al. (2015), it can however produce a very different MDF, which depends to first order on the shape of the SFH. Figure 1 shows the MDF, age-metallicity relation, and corresponding SFH assuming a CBM obtained in Snaith et al. (2015; thick curve). The only model constraint was to best fit the age- $[\mathrm{Si} / \mathrm{Fe}]$ relation of solar vicinity stars, which as we argued in Haywood et al. (2015) should be representative of the whole inner disk. The MDF shows two distinct peaks at $[\mathrm{Fe} / \mathrm{H}]=+0.3$ and -0.2 dex, separated by a dip at $[\mathrm{Fe} / \mathrm{H}]=+0 \operatorname{dex}($ or $[\mathrm{Fe} / \mathrm{H}]=+0.1$ dex for the smoothed $\mathrm{SFH})$, which corresponds to the epoch when the MW, in a similar way to other galaxies, quenched its star formation activity according to Haywood et al. (2016a), as can be seen by the shaded areas in the three plots. The peak at $[\mathrm{Fe} / \mathrm{H}]=-0.2 \mathrm{dex}$ is the signpost of the thick disk formation and the peak at $[\mathrm{Fe} / \mathrm{H}]=+0.3$ dex corresponds to the stars that formed during the (inner) thin disk phase (age $<7.5 \mathrm{Gyr}$ ). The smoothed model shows that the third peak of the MDF at $[\mathrm{Fe} / \mathrm{H}] \sim-0.8$ dex is due to the small peak in the SFH at age $\sim 12 \mathrm{Gyr}$, which is within the uncertainties of the SFH and cannot be taken as significant. 

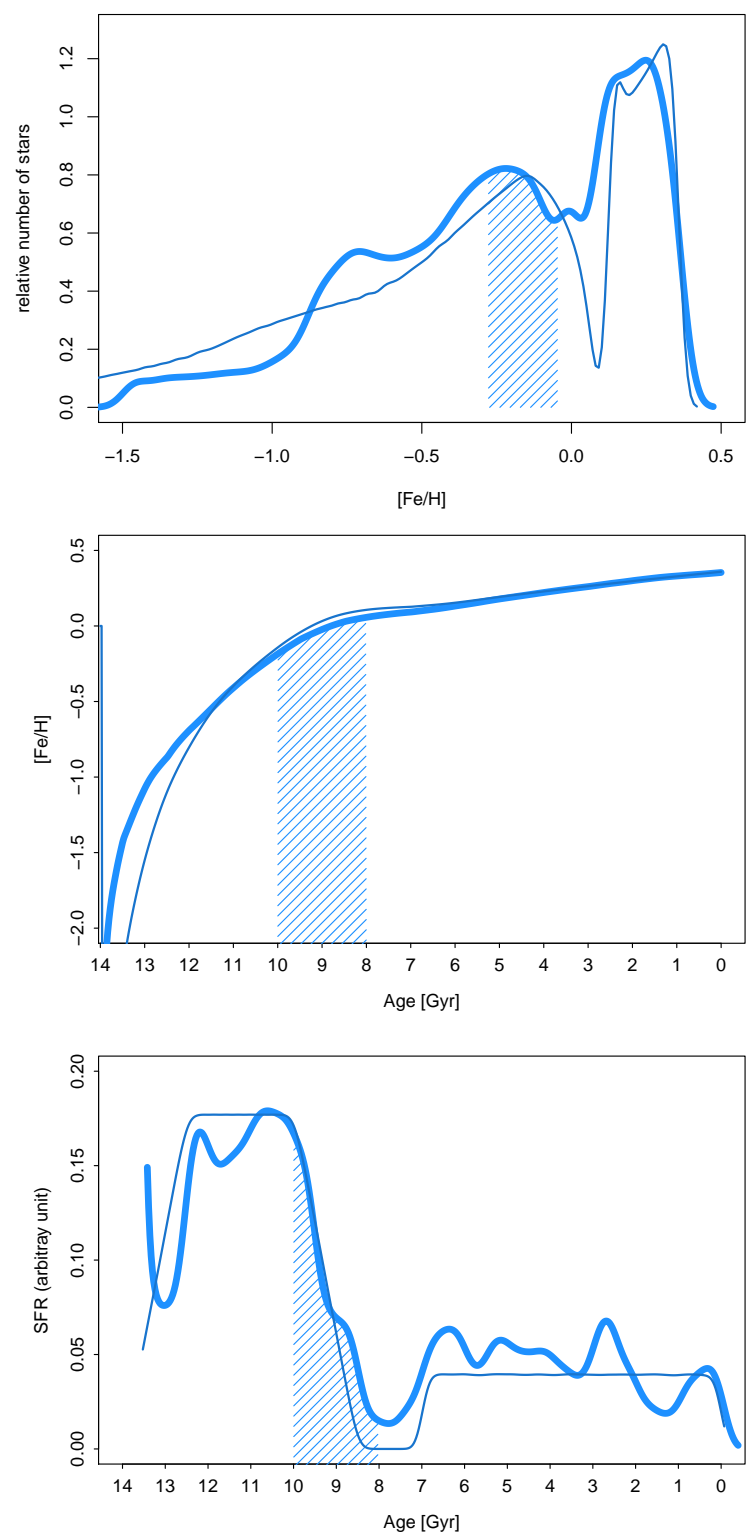

Fig. 1. Distributions of our inner disk model. Bottom panel: SFH middle: age-metallicity relation; top: metallicity distribution. The thick curve corresponds to the best fit model derived in Snaith et al. (2015), or "fiducial model", while the thin curve represents the same model, but where the small-scale variations in the SFH have been smoothed out. It shows in particular that the third peak (at $[\mathrm{Fe} / \mathrm{H}] \sim-0.8 \mathrm{dex}$ ) in the Snaith et al. (2015) is due to the small peak in the SFH at age $\sim 12 \mathrm{Gyr}$, and is probably not significant. The blue area in each plot emphasizes the quenching epoch of the SFR in the MDF and age-metallicity relation.

We note that the present model is entirely constrained by fitting the inner disk sequence of the solar vicinity age- $[\mathrm{Si} / \mathrm{Fe}]$ relation (Snaith et al. 2015), and that it is not tuned in any aspect to fit the in-situ inner disk and bulge data. We do not introduce any additional parameters, such as a radially dependent star formation timescale (as a consequence of a radially dependent accretion timescale; e.g., Minchev et al. 2014; Kubryk et al. 2015a) or radial migration, which are essentially unconstrained, and therefore are defacto free parameters. We also emphasize that the model presented here applies to the inner disk only: the outer disk and solar vicinity have different age- $[\alpha / \mathrm{Fe}]$, age- $[\mathrm{Fe} / \mathrm{H}]$ relations and MDFs from the inner (thick + thin) disks and need separate chemical evolution modeling (see Snaith et al. 2015 for the modeling of the outer thin disk age- $[\alpha / \mathrm{Fe}]$ relation). A scenario for the chemical evolution of the solar vicinity is presented in Haywood et al. (in prep.).

\subsection{Ingredients}

The ingredients of our model are described and discussed at length in Snaith et al. (2015). The standard theoretical yields are from Iwamoto et al. (1999), Nomoto et al. (2006), and Karakas (2010), the IMF from Kroupa (2001). The stellar mass-lifetime relation is dependent on the metallicity and is taken from Raiteri et al. (1996). The time delay function is taken from Kawata \& Gibson (2003). As mentioned above, no instantaneous recycling approximation has been assumed; see Snaith et al. (2015) for details.

\section{Results: Chemical evolution of the inner disk}

\subsection{Data and the metallicity distribution function of the inner disk from APOGEE}

The Apache Point Observatory Galactic Evolution Experiment (APOGEE; Majewski et al. 2017) is a near-infrared, highresolution $(R \approx 22500)$ spectroscopic survey of stars in the MW, included as part of the third and fourth Sloan Digital Sky Survey. In the present study, we use the data release 13 of the APOGEE survey. Starting from a sample of $\sim 104000$ stars in APOGEE with distances (and $\sim 15000$ within $R<6 \mathrm{kpc}$ ) from Wang et al. (2016), we select APOGEE objects as recommended in the DR13 documentation, discarding objects with $T_{\text {eff }}>5250 \mathrm{~K}$ or $[\mathrm{Fe} / \mathrm{H}]<-1$ dex or outside the interval $1.0<\log g<3.8$. Within these limits, it has been shown by Hayden et al. (2015) that the sampling in directions, magnitudes, and colours of APOGEE does not introduce any significant bias in the metallicity distribution function of the survey. Giants however probably bias the underlying age distribution against the oldest objects, or the most alpha-rich stars; see Bovy et al. (2014). Therefore, we keep in mind that the survey possibly underestimates the relative number of old stars compared to younger ones, or the number of stars in the thick disk.

Figure 2 shows the $[\mathrm{Fe} / \mathrm{H}]-[\alpha / \mathrm{Fe}]$ distributions of the APOGEE data from 3 to $6 \mathrm{kpc}$ from the Galactic center in three different distance intervals, assuming the Sun is at $8 \mathrm{kpc}$ from the Galactic center. $[\alpha / \mathrm{Fe}]$ is defined by the mean of $\mathrm{Mg}$ and $\mathrm{Si}$ abundance ratios. A finite mixture density estimation in this plane (using the Mclust $\mathrm{R}$ package) indicates two main components around the following mean metallicities and alpha abundances in the three distance intervals, 5-6, 4-5, $3-4 \mathrm{kpc}:[\mathrm{Fe} / \mathrm{H}]=-0.40,-0.41,-0.35,[\alpha / \mathrm{Fe}]=0.20,0.19,0.20$, for the metal-poor component and $[\mathrm{Fe} / \mathrm{H}]=0.16,0.17,0.12$, and $[\alpha / \mathrm{Fe}]=0.018,0.017,0.028$, for the metal-rich. These values are near the centroids of the two components seen in the density contour plots (Fig. 2). The first two distance bins (5-6/4-5 kpc) show additional components at $[\mathrm{Fe} / \mathrm{H}]=-0.11,-0.06 \mathrm{dex}$, and $[\alpha / \mathrm{Fe}]=0.05,0.05 \mathrm{dex}$, representing respectively $24 \%$ and $18 \%$ of the sample, which we interpret as contamination by solar vicinity/outer-disk stars. This third component is not found in the third bin $(3-4 \mathrm{kpc})$. In order to limit the contamination by this last component, we selected stars as in Haywood et al. (2016a), keeping objects above our standard model lowered by 0.05 dex (black curve). The resulting MDFs are shown on the lower plots as gray histograms. This selection limits, but cannot completely eliminate, contamination by solar vicinity/outer-disk stars, 

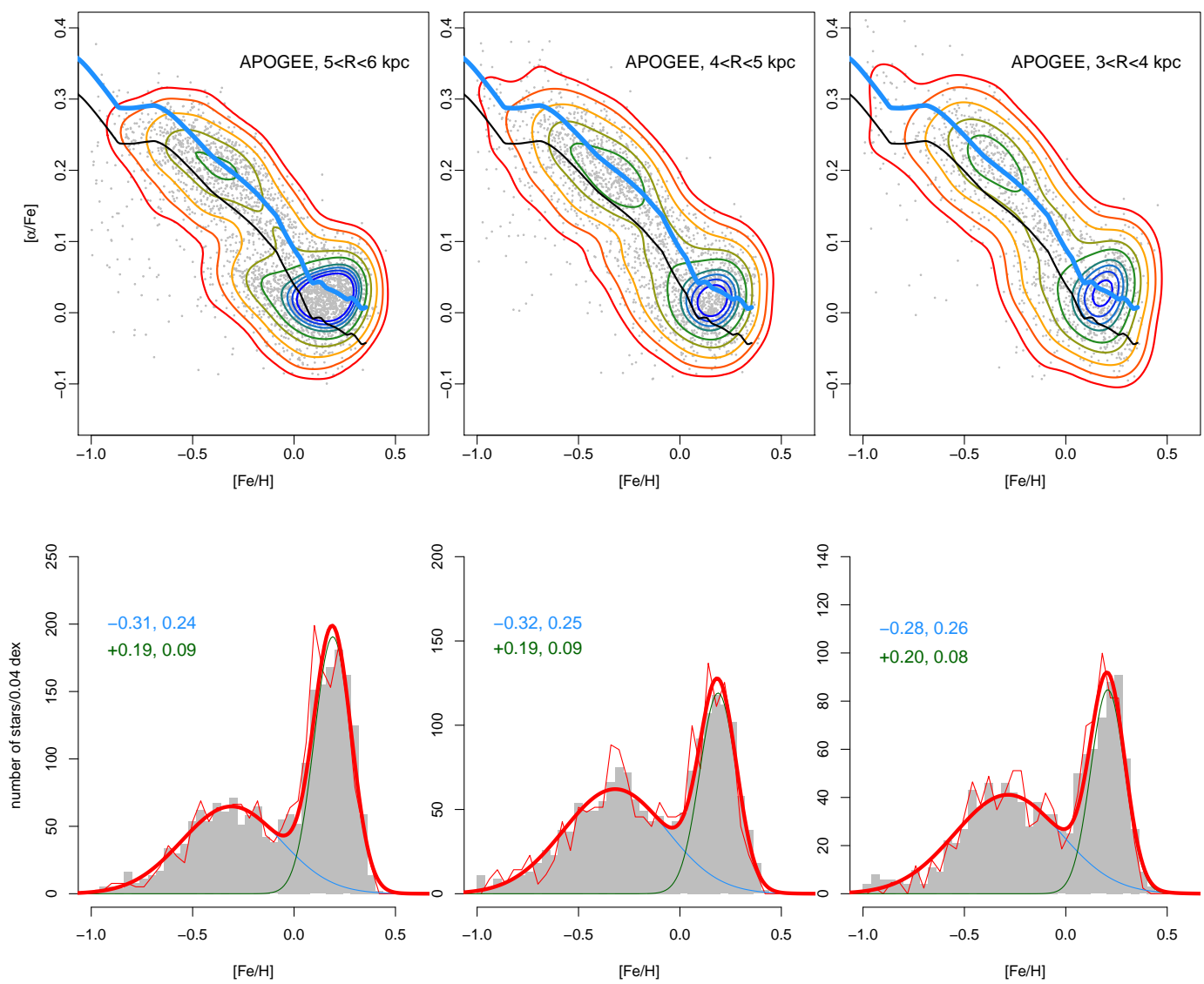

Fig. 2. Top: $[\alpha / \mathrm{Fe}]-[\mathrm{Fe} / \mathrm{H}]$ distribution of stars from APOGEE in different distance bins as indicated on each plot. Our standard model is shown in blue, the same model lowered by -0.05 dex in black. Stars that compose our MDFs are selected above this line. Bottom: gray histograms are MDFs of stars selected above the black line. The thick red curves are the sum of the two components (blue and green) in the finite mixture decomposition applied to the gray histograms. The mean metallicity and dispersion of each component is given on each plot. The red thin curves are the histograms obtained by selecting stars in the APOGEE catalog with a signal-to-noise ratio (S/N) above 150 (instead of 50 for the gray histogram).

particularly in the first distance bin, between 5 and $6 \mathrm{kpc}$. We have chosen not to use the estimated membership probabilities of individual stars given by Mclust because the third component, dominated by outer-disk objects, also comprises a number of stars which are clearly on the inner-disk sequence. Removing them would artificially enhance the bimodality of the distributions.

We also applied the finite mixture density estimation to one-dimensional metallicity histograms (gray histograms, bottom plots of Fig. 2) obtained after the selection of our stars, searching for two and three components. The Bayesian information criterion (BIC) defined by $\mathrm{BIC}=-2 \ln (L)+k \ln (n)$ (where $L$ is the likelihood of the model, $n$ is the number of bins and $k$ the number of parameters to assess the goodness of the fit), shows that in all three distance bins, the data is fitted equally well with two or three components. For the first and second distance bins, it is either the main metal-rich or the metalpoor peaks which are sub-divided in two components, and the BIC information is inconclusive, slightly favoring three components in the first distance bin, and two in the second one. In the third distance bin, the third component is only $2 \%$ and has a mean metallicity of -0.91 . Hence, the two-component fit offers a more uniform solution, with the mean metallicities of the metal-poor $([\mathrm{Fe} / \mathrm{H}]=-0.30 \pm 0.02 \mathrm{dex})$ and the metalrich $([\mathrm{Fe} / \mathrm{H}]=0.19 \pm 0.01 \mathrm{dex})$ components very similar in all distance bins and dispersions which are about +0.25 dex for the metal-poor component and 0.09 dex for the metal-rich. These parameters are very similar to the maximum of the density contours shown in the $[\mathrm{Fe} / \mathrm{H}]-[\alpha / \mathrm{Fe}]$ distribution, and to the parameters of the two main components found in the $2 \mathrm{D}$ analysis above, with only a limited increase in the mean metallicity of the metalpoor component by about $+0.05-0.1$ dex. Hence, our selection procedure keeps intact the main characteristics of the $2 \mathrm{D}$ distributions. In all distance intervals, the transition between the metal-poor and metal-rich peaks is found at solar metallicity. This is very similar to the result obtained by several groups on the bulge, where two dominant peaks are most often found; see for instance Zoccali et al. (2017). In all plots, stars have been selected with a minimum $S / N$ of 50 . We checked that changing the limit does not significantly affect the shape of the MDF, apart from increasing the Poisson noise in the histogram. The effect of setting the $S / N$ limit to 150 is shown on each plot as red curves. As can be seen, the change is minimal.

\subsection{The metallicity, $[\alpha / \mathrm{Fe}]$ and $[\alpha / \mathrm{H}]$ distribution functions}

Figure 3 shows the comparison between the APOGEE distribution of metallicity (left column), $[\alpha / \mathrm{Fe}]$ (middle), and $[\alpha / \mathrm{H}]$ (right) of the selected objects together with the predictions of the model shown in Fig. 1 in three different radial bins. 

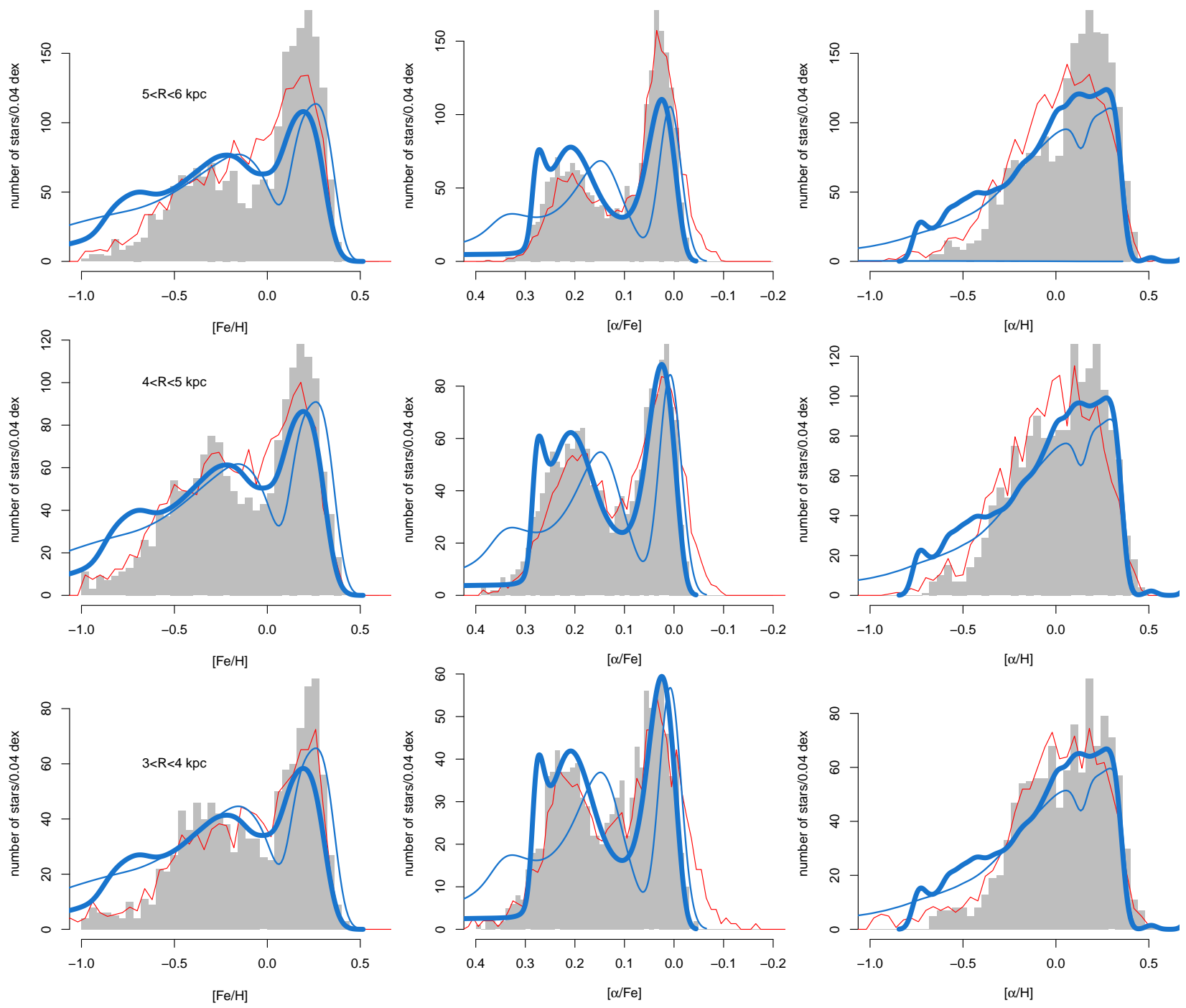

Fig. 3. Comparisons between the model and APOGEE data in different radial bins, as indicated on the left plots. From left to right: MDF, $[\alpha / \mathrm{Fe}]-\mathrm{DF}$, and $[\alpha / \mathrm{H}]-\mathrm{DF}$. The APOGEE data with and without the selection in the $[\mathrm{Fe} / \mathrm{H}]-[\alpha / \mathrm{Fe}]$ plane are shown as gray histograms and red curves. Red curves are normalized to the gray histograms. The blue thick curve is our fiducial model, the blue thin curve is the smoothed model. Both models are normalized to have the same number of stars as the data.

The first thing to note is the difference in the shape of the observed MDF in the inner disk and at the solar vicinity (and the difference is visible starting already at $R \lesssim 7 \mathrm{kpc}$ ). The former reflects the bimodality seen in the $[\alpha / \mathrm{Fe}]$ distribution, which we discussed in Haywood et al. (2016a) and is also clearly seen on the $[\mathrm{Fe} / \mathrm{H}]-[\alpha / \mathrm{Fe}]$ distributions (lower plots of Fig. 2), while the latter peaks at solar metallicity and is unimodal (e.g., Haywood 2001). The MDFs in the inner disk are clearly bimodal, with peaks at metallicities $[\mathrm{Fe} / \mathrm{H}] \sim-0.30$ and +0.25 dex, corresponding to the thick disk and the thin (inner) disk, respectively, two populations that are in minority at the solar radius, while there is clearly a dip at solar metallicity. The rise of these two populations in the inner disk is well in line with the small-scale lengths measured by Bovy et al. (2016) on the APOGEE data for the alpha-enhanced and the metal-rich populations.

Our model is also shown on each plot, normalized so as to have the same number of stars as in the observations. We emphasize that these are not fits, but are entirely derived from the SFH deduced by fitting the model to the age- $[\mathrm{Si} / \mathrm{Fe}]$ of the inner disk as observed in the solar vicinity, so there is no adjustment whatsoever on any radially dependent parameter (radial migration, infall timescale, etc.). For this reason, we consider that our fiducial CBM is a satisfactory first representation of the data, capturing the main features of all three distributions in all three distance bins: the width of the distribtions, the position of the two main peaks, and the presence of the dip in the MDFs and $\alpha$ DFs. Obviously, there is also room for adjustment. For example, the errors in the abundances are uncertain. While we convolved our models with an uncertainty of 0.05 dex on metallicities using the estimate from Bovy et al. (2016), individual internal errors in the catalog are peaked at about 0.02 dex. Second, the bump at $[\mathrm{Fe} / \mathrm{H}] \sim-0.8$ dex in our model is due to a small variation in the SFH and is not significant, but it remains however that both our fiducial and the smoothed models are overpredicting the number of stars below this metallicity. There may be several reasons for this offset. Firstly, giant tracers favor younger stars and possibly underestimate older (alpha-rich) objects; see Bovy et al. (2014). We do not expect this bias to affect only stars at $[\mathrm{Fe} / \mathrm{H}]<-0.7 \mathrm{dex}$, because all stars older than 4-5 Gyr may be affected; thick disk objects most significantly. However, if 
we had normalized our fiducial model on the metal-rich peak (instead of the total number of stars), it would be apparent then that the model slightly overpredicts the number of thick-disk stars $([\mathrm{Fe} / \mathrm{H}]<0 \mathrm{dex})$, and this could be explained by the bias induced by giant tracers.

The second reason that comes to mind is the fact that the "instantaneous accretion" model - that is, the CBM - is a (too) rough approximation of a situation where accretion occurs rapidly. Stars at $[\mathrm{Fe} / \mathrm{H}]<-0.7$ dex have ages of $>12 \mathrm{Gyr}$. It is very well possible that a residual "G-dwarf problem" remains at these metallicities/ages. If the accretion was fast (but not instantaneous, as modeled here), the number of stars at ages $>12$ Gyr and $[\mathrm{Fe} / \mathrm{H}]<-0.7$ dex would be less than predicted as in the standard "G-dwarf problem", shifted to lower metallicities. Taking into account a small (but not zero) accretion time at ages $>12$ Gyr would probably improve the comparison between the models and the data, in a similar way to how standard infall models are "solving" the G-dwarf problem at higher metallicities.

Comparisons between the APOGEE $[\alpha / \mathrm{Fe}]$ distribution function (DF) and our standard model have already been shown in Haywood et al. (2016a); we add here our smoothed model. The comparisons between the APOGEE $[\alpha / \mathrm{H}]$-DF and the models are given in the right column of Fig. 3. Because iron is absent from these plots, the comparisons are independent of the modeling of the iron production and in particular the time-delay function used for the SNIa.

To conclude, the main features of the MDF, $[\alpha / \mathrm{Fe}]-\mathrm{DF}$ and $[\alpha / \mathrm{H}]-\mathrm{DF}$, are reproduced by our model with the exception of the offset at the metal-poor end, as previously discussed.

We emphasize that the aim here is not to fine-tune the comparisons and to design a best fit model, but to check how, in first approximation, a CBM fitted on inner disk stars found in the solar vicinity is capable of representing the main observed features of chemical abundances. Reproducing the details of observed distributions is also less meaningful given that the APOGEE data has its own uncertainties (contamination by outer-disk solar vicinity stars and the distances, among others), while the MDF as a function of $R$ is likely to improve significantly in the near future, with Gaia DR2 in particular.

\subsection{The age-metallicity relation}

The most difficult constraints to obtain at the moment are those depending on ages, and these are still limited to the strict solar vicinity. Our only possibility is therefore to select "inner disk" stars from a solar vicinity sample, and we use the sample of Adibekyan for which we have age measurements (Haywood et al. 2013, 2015). Figure 4a shows the sample of stars with ages from Haywood et al. (2013) and metallicities from Adibekyan et al. (2012). To select inner-disk stars more accurately, we keep the stars that are above the black curve in plot (a), which is the model representing the data (blue curve) lowered by 0.025 dex. We impose a stricter limit than for stars at $R<6 \mathrm{kpc}$, because the contamination at the solar vicinity is likely to be more important. This selection is likely to be accurate for the part of the inner disk sequence well separated from the local stars, which is true for $[\alpha / \mathrm{Fe}]>+0.05$ dex, or ages of $>7$ Gyr. Below this limit the contamination by local stars will increase. For the stars selected as inner disk objects, color codes the age according to the vertical scale on the right of the plot. The three larger circles represent the young, alpha-rich stars identified in our sample (Haywood et al. 2013, 2015), while the black triangle represents the position of the open cluster NGC 6791 in all three plots.
Plot (b) shows the age-metallicity relation for the whole sample and for the inner disk stars. The dispersion increases slightly at ages $<5$ Gyr because our selection of "inner disk" stars at $[\alpha / \mathrm{Fe}]<0.05$ dex most likely includes "OLR" stars, which have lower metallicities at a given age. At even lower metallicities $([\mathrm{Fe} / \mathrm{H}]<-0.2 \mathrm{dex})$ and ages $<7 \mathrm{Gyr}$, the three young alpha-rich stars are also visible. Otherwise, we note that the age-metallicity relation is in good agreement with the model.

Figure 4 shows two important results: (1) when inner disk stars are selected, the dispersions in chemical abundances and metallicities at a given age are very small, both on the agemetallicity and age- $[\alpha / \mathrm{Fe}]$ plots. This means that, contrary to what is observed at the solar vicinity, where the spread in metallicity at all ages is observed, samples of in-situ inner-disk stars should show tight age-chemistry relations, or small dispersion at all ages. This point is discussed further in Sect. 5. (2) The second result is that the evolution of the inner thin disk (ages $<7 \mathrm{Gyr}$ ) is in continuity with that of the thick disk, and corresponds to the upper envelope of the age-metallicity distribution observed at the solar vicinity. Within the OLR, the thin disk started to form stars from the conditions left by the thick disk after the quenching episode, and continued a monotonic enrichment, illustrated by the model, with no apparent dilution.

The OLR, by establishing a barrier limiting the inflow of gas in the inner disk, presumably permitted a monotonic enrichment described by the CBM. At the OLR and beyond, the ISM left by the thick disk evolution may have mixed with lower metallicity gas present in the outer disk, explaining why stars at any given age at the OLR and beyond have a metallicity lower than innerdisk stars. As mentioned in Haywood et al. (2013), the lowermetallicity gas coming from the outer disk could have been polluted by metals during the thick-disk phase.

To summarize, we find that our model is a good representation of the age- $[\alpha / \mathrm{Fe}],[\mathrm{Fe} / \mathrm{H}]-[\alpha / \mathrm{Fe}]$, age-metallicity, and metallicity distributions of the inner disk. Haywood et al. (2016a) have also shown that the model satisfactorily reproduces the $[\alpha / \mathrm{Fe}]$ distributions of the APOGEE survey in the distance range considered here. To our knowledge, it is the first time that a Galactic chemical evolution model provides a good match to such a large range of observational constraints of the inner disk.

The case of NGC 6791. Also added to Fig. 4 are the positions of the metal-rich cluster NGC 6791. This cluster is often presented as an outlier to the chemical evolution of the disk (Carraro et al. 2006; Geisler et al. 2012; Carraro 2014). However, with age estimates between $6.8 \pm 0.4$ and 8.6 $\pm 0.5 \mathrm{Gyr}$ (Basu et al. 2011), and a metallicity of $\sim+0.3 \mathrm{dex}(+0.3 \pm 0.02 \mathrm{dex}$, Boesgaard et al. 2015, +0.29 dex, Brogaard et al. 2011), NGC 6791 is typical of the evolution of the inner disk, as described here. The alpha element abundance of NGC 6791, as deduced from the study of Boesgaard et al. (2015) is +0.036 (taking the mean of $\mathrm{Mg}$, $\mathrm{Si}$ and $\mathrm{Ti})$, and falls well on both the observed age- $[\alpha / \mathrm{Fe}]$ and $[\mathrm{Fe} / \mathrm{H}]-[\alpha / \mathrm{Fe}]$ relations; see Fig. 4 . If the age of NGC6791 is confirmed to be greater than $7 \mathrm{Gyr}$, this implies that it would have formed during the quenching episode that occurred in the MW at this time (Haywood et al. 2016a). Both our SFH, taken as a birth probability function (and assuming that our age scale is correct), and the age-metallicity relation favor a slightly lower age determination.

Jílková et al. (2012) tried to reconcile the present orbit of NGC $6791\left(R_{\text {peri }}=5-5.3 \mathrm{kpc}, R_{\text {apo }}=8.5-9.1 \mathrm{kpc}\right)$ and eccentricity in the range $0.23-0.29$ with the eccentricity defined as $\left(R_{\text {apo }}-\right.$ $\left.R_{\text {peri }}\right) /\left(R_{\text {apo }}+R_{\text {peri }}\right)$ with a scenario where the cluster originated in the inner disk $(R<5 \mathrm{kpc})$, possibly down to $R \sim 3 \mathrm{kpc}$, and then moved outwards by radial migration. With the properties of 

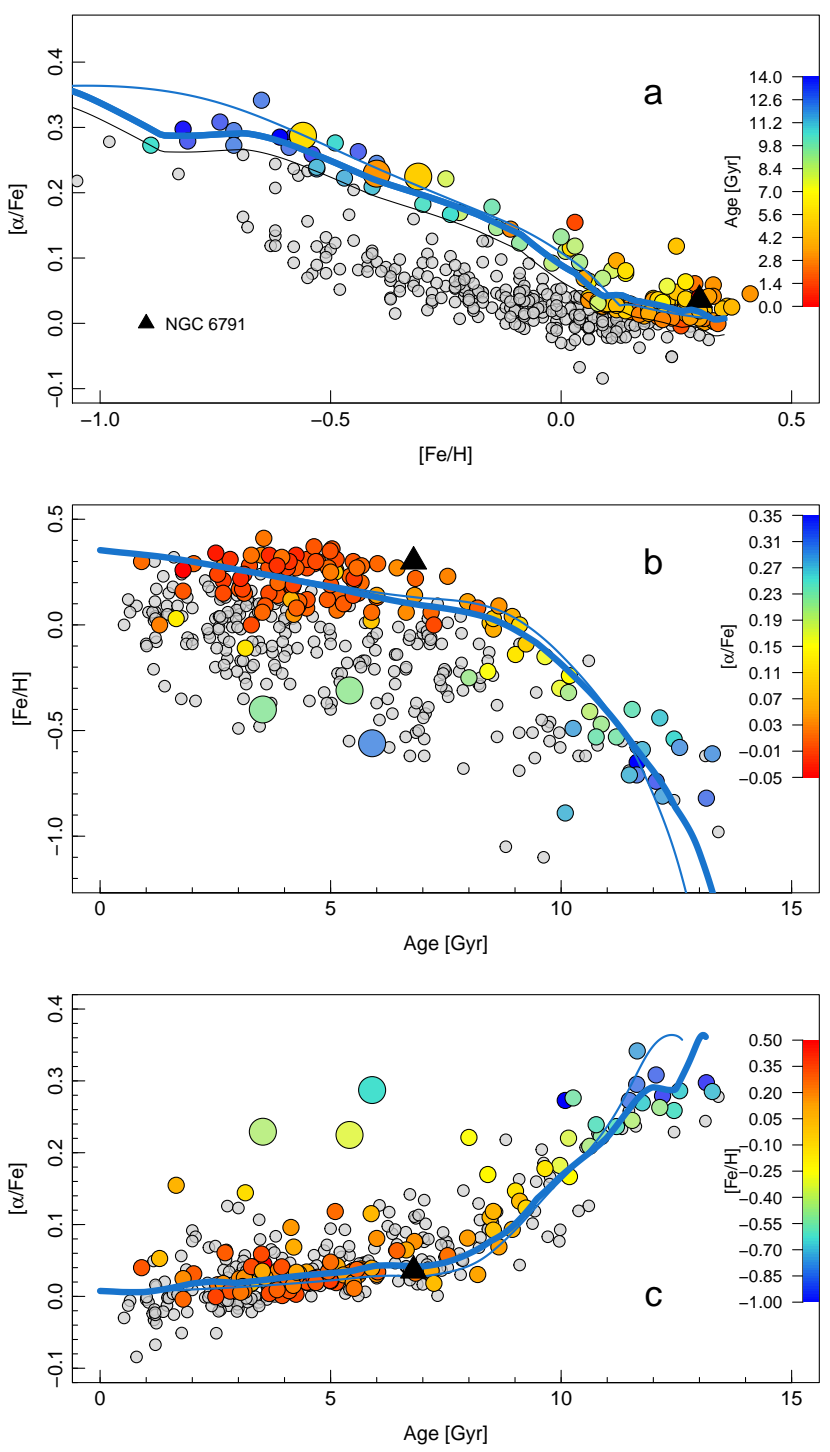

Fig. 4. Panel a: selected inner disk stars from the sample of Adibekyan et al. (2012), together with our models (blue curves). The stars are selected above the fiducial model lowered down in $[\alpha / \mathrm{Fe}]$ by 0.025 dex (black curve) in the $[\mathrm{Fe} / \mathrm{H}]-[\alpha / \mathrm{Fe}]$ distribution. The color scale codes the age of the stars from Haywood et al. (2013). Panel $b$ : age-metallicity distribution of inner-disk stars described in the text. The observed distribution widens at younger age $(<5 \mathrm{Gyr})$ because of the increasing difficulty in separating the inner disk sequence from the OLR (local) stars at lower alphas. Panel $c$ : age- $[\alpha / \mathrm{Fe}]$ relation for the selected stars, together with the model. The three points with larger symbols are apparently young, alpha-rich objects. The black triangles indicate the position of NGC 6791 at age $=6.8 \mathrm{Gyr},[\mathrm{Fe} / \mathrm{H}]=+0.3$ and $[\alpha / \mathrm{Fe}]=+0.036$ (see text for references).

the inner disk described here however, the cluster does not have to originate from Galactocentric radii as small as $3 \mathrm{kpc}$. If the cluster was born within $6-7 \mathrm{kpc}$ of the Galactic center, it would still be fitted to its local environment since the mean metallicity of the thin disk in this radial bin is about +0.25 dex. NGC 6791 is the best example of a metal-rich object that can pollute the solar orbit by having eccentricities sufficiently high that blurring alone is enough to explain their presence at the solar circle. The study of Jílková et al. (2012) is also a nice illustration of the result found by Hallé et al. (2015), showing that the OLR of the bar plays the role of a barrier for the migration of stars. Figure 4 of Jílková et al. (2012) shows that this is true not only when the bar is the only asymmetry, but also when there are spiral arms, or both a bar and spiral arms. In all cases, the cluster has a negligible probability to cross the OLR of the bar. We note that this effect is also nicely confirmed by Monari et al. (2016), who also show that the OLR acts as a strict barrier to the stars when the bar is the sole asymmetric perturbation (their Fig. 11, left plot), while their simulation shows that even when there is a coupling between the bar and the spiral arms, the extent of radial mixing is extremely limited $(<500 \mathrm{pc}$ for most of the stars; $<1 \mathrm{kpc}$ for essentially all the stars).

\section{Results: Chemical evolution of the bulge}

If the majority of the bulge is made of material transferred from the disk, we expect the stars of the bulge to have stellar ages and chemical characteristics (both the global MDF and abundance ratios) not different from that of the disks. The chemical evolution of the MW bulge is commonly modeled as an independent system, even when it is acknowledged that part of its structure and content must have come from the disk through dynamical instabilities (e.g., Grieco et al. 2012; Tsujimoto \& Bekki 2012). This is because of two properties, often thought to be the landmark of the bulge:

(1) The MDF of the bulge is very broad, reaching both high and low metallicities, and has a minimum at solar metallicity, while the disk MDF, as sampled in the solar vicinity, is narrower and peaks at solar metallicity. This is often presented as evidence that the bulge origin is not in the disk (see e.g., McWilliam 2016, Fig. 3 ). We argue below that since only stars whose guiding radii are within the OLR participate in the bar, comparing the bulge and solar vicinity MDFs is incorrect, while, as we show below, the MDF of the inner disk and the MDF of the bulge are compatible.

(2) The bulge seems to be uniformly old (>10 Gyr). Reaching the high metallicities $([\mathrm{Fe} / \mathrm{H}]>+0.4 \mathrm{dex})$ observed in the bulge within 3-4 Gyr after the Big-Bang is challenging, and requires a particular chemical evolution. Recent works however suggest that the bulge is not uniformly old. Bensby et al. (2011, 2013) show that the age spread in the metal-rich component of the disk is at least $\sim 10 \mathrm{Gyr}$, with stars that have ages as low as $2 \mathrm{Gyr}$ (see also Groenewegen \& Blommaert 2005; Catchpole et al. 2016; Haywood et al. 2016b; Schultheis et al. 2017).

Allowing the bulge to have much younger stars than previously thought releases the difficulty of having to design models capable of reaching high metallicities within a few Gyr.

We now explore in more detail how our model matches the bulge chemical characteristics.

\subsection{The bulge metallicity distribution function}

Perhaps the most significant hurdle in identifying the bulge population with the thick and thin disks is the MDF of the bulge, which is much broader than the disk MDF measured at the solar vicinity. As mentioned in the introduction, stars are driven to the inner regions to form the bar through dynamical instabilities from regions inside the OLR, as quantified in detail in Di Matteo et al. (2014), Hallé et al. (2015) and Hallé et al. (2018). In the MW, the OLR resonance is located near the solar circle (7-10 kpc) according to Dehnen (2000) and Pérez-Villegas et al. (2017). This implies that, if the bulge is essentially made of disk stars, it must be dominated by inner disk $(R \lesssim 7 \mathrm{kpc})$ objects, and not by the kind of thin-disk stars that dominate the solar vicinity and beyond. Di Matteo et al. (2014, 2015) explicitly identified populations A, B and C of Ness et al. (2013b) as thick disk (for C and B) and inner thin disk (for 
population A). Fragkoudi et al. (2017) showed that this scenario is able to reproduce the mean metallicity map of the bulge, as revealed by APOGEE. We thus expect the (global) MDF of the bulge to be the same as the MDF of the inner disk. There are two caveats here. The first is that we lack a measurement of the global MDF of the bulge, having only estimates of the MDF in different limited regions. Hence, one must chose a distribution that is representative of the global MDF. Second, because stars are redistributed in the bulge according to their initial kinematics (i.e., thick or thin disks) and initial location in the disk, the MDF will vary as a function of latitude and longitude.

For instance, Di Matteo et al. (2014) have found that stars originating closer to the OLR tend to populate the outer region of the bulge. Most importantly, the relative fraction of thin and thick disk stars is a strong function of latitude, with the metal-rich thin disk dominating at low latitude and the metal-poorer thick disk at high latitude (except along the bulge minor axis, at very low latitudes, where this trend seems to invert; see Zoccali et al. 2017). Therefore, we do not expect the observed MDF sampled at any given location in the bulge to be closely similar to the disk MDF. Since we do not model the spatial distribution of these two populations in the bulge, the comparison of the model and observed MDF can only be qualitative, with attention to the most important features rather than to details. We refer to Fragkoudi et al. (2018) for a detailed modeling of the MDF of the bulge as a function of longitude and latitude.

Figure 5 (top) shows the MDF of our model (blue curve) together with the MDF from the ARGOS survey at $b=-5^{\circ}$ (Ness et al. 2013b) and with the MDF from Bensby et al. (2013; middle plot). The distribution from Bensby et al. (2013) is made of stars that are mostly within latitudes $-6^{\circ}<b<-2^{\circ}$ and longitudes $-6^{\circ}<l<7^{\circ}$, while the MDF from Ness et al. (2013b) is made of a series of fields at different longitudes, in the interval $-15^{\circ} \leq l \leq+15^{\circ}$ but limited to $b=-5^{\circ}$. Also, the MDF from Ness et al. (2013b) contains several thousand stars, while the sample from Bensby et al. (2013) contains 58 dwarfs and subgiant stars observed through microlensing events. While observed MDFs in the galactic plane would favor the contribution of the thin disk (the metal-rich peak), those out of the plane favor the thick disk (the metal-poorer peak). Hence, the field from Ness et al. (2013b) at $b=-5^{\circ}$ is unlikely to severely underestimate one of the two, but however systematic differences with the model MDF are not unlikely, and the sampling at this particular latitude may not represent the ratio of the thin and thick disks of the model - that is the observed ratio between the metal-rich to metal-poor peak.

In spite of these differences, the two agree fairly well, and in particular they both show the dip in the MDF at $[\mathrm{Fe} / \mathrm{H}]=0$ dex. The difference between Ness et al. and Bensby et al. at supersolar metallicities illustrates that the metallicity scale, in particular at $[\mathrm{Fe} / \mathrm{H}]>0$ dex, is not secure, and can vary significantly from one study to the other ${ }^{1}$. Bottom plot compares the models with MDFs from Zoccali et al. (2017) at two latitudes, $-3.5^{\circ}$ and $-6^{\circ}$ and which combine a range of longitudes (from -8 to $+8^{\circ}$ ), using the Gaussian components given in their Table 3. Each MDF combines samples at different longtitudes (their Fig.7). These two latitudes were chosen for the same reason that we chose ARGOS field at $b=-5^{\circ}$ : the

\footnotetext{
1 As another example, Rojas-Arriagada et al. (2014) have found a systematic offset between their metallicities and those of Hill et al. (2011) which amounts to +0.21 dex, for a subset of one hundred stars common to both studies. Once the MDF from Hill et al. (2011) is shifted to lower metallicities by this amount, the agreement between our model and their MDF is also satisfactory; see Haywood et al. (2016b).
}
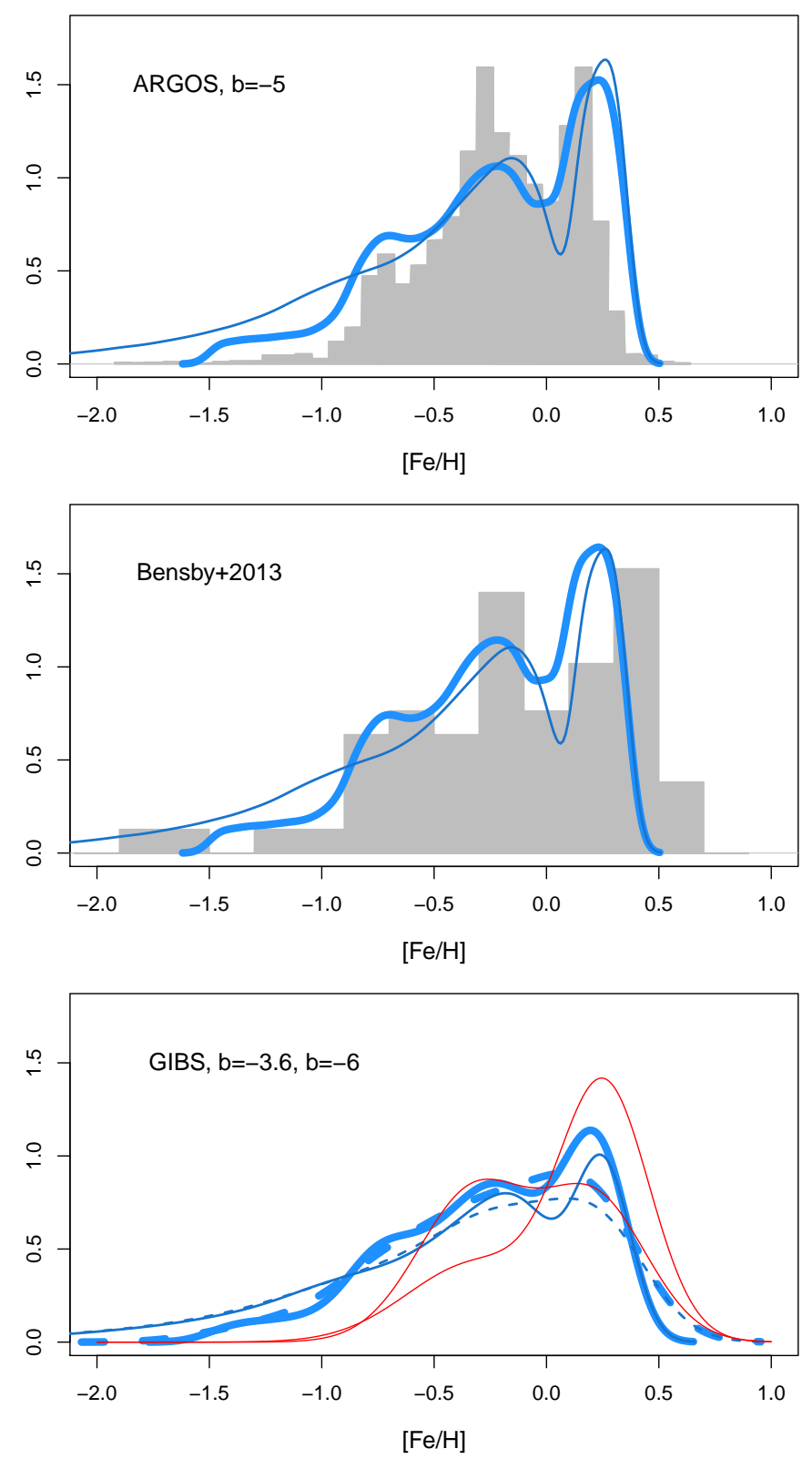

Fig. 5. Comparison of the fiducial model MDF (thick blue line) and smoothed model (thin blue line) with different observed bulge MDFs from Ness et al. (2013b, top), Bensby et al. (2013, middle) and Zoccali et al. (2017). Two MDFs at different latitudes Zoccali et al. (2017) are shown, $b=-3.6$ and $-6^{\circ}$, and in this case models have been convolved with a Gaussian of $0.1 \mathrm{dex}$ (continuous blue curves) and 0.2 dex (dashed blue curves).

spatial segregation at theses latitudes will not overly favor either metal-poor or metal-rich populations. According to Zoccali et al. (2017), the errors in metallicities range from 0.1 to 0.4 dex (from metal-poor to metal-rich stars), with a mean at 0.2 dex. To take this into account, we convolved our standard model with Gaussian with two dispersions, 0.1 and 0.2 dex, represented by the thick continuous and dashed curves. The two observed MDFs illustrate the significant variability between two MDFs separated by only $\sim 350 \mathrm{pc}$ in the vertical direction, with the metal-rich component representing $67 \%$ of the stars in the field at $3^{\circ}$, and $20 \%$ less at $6^{\circ}$ (Zoccali et al. 2017).

Figure 6 shows the comparisons with the APOGEE survey. The stars from APOGEE representing the bulge have been 

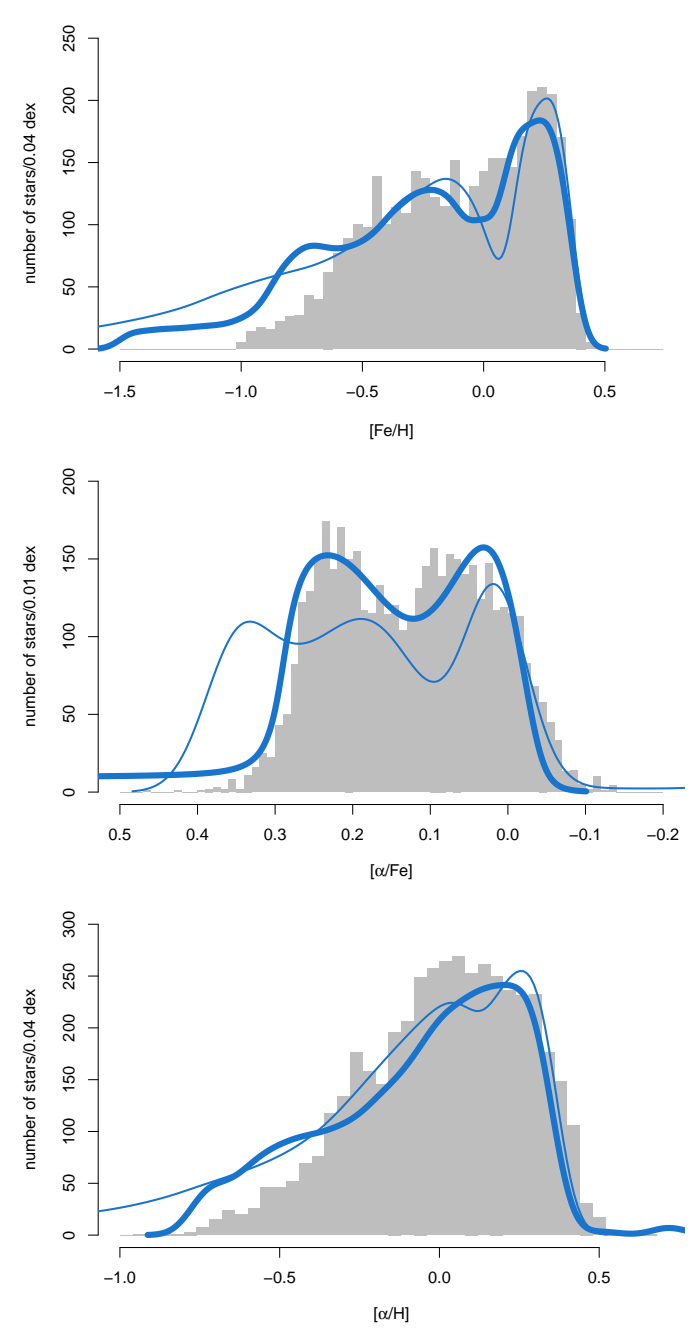

Fig. 6. APOGEE sample of stars within $3 \mathrm{kpc}$ of the Galactic center, chosen to represent the bulge population, compared with our models. The models are normalized to have the same number of stars as the data in the metallicity range $[\mathrm{Fe} / \mathrm{H}]=-1$ to $+0.5 \mathrm{dex}$. Thin curve: smoothed model. Thick curve: fiducial model. Upper plot: comparison of the metallicity distributions. Middle plot: comparison of the $[\alpha / \mathrm{Fe}]$ distributions. Lower plot: $[\alpha / \mathrm{H}]$ distributions.

selected within $3 \mathrm{kpc}$ from the Galactic center (longitudes between 0 and $20^{\circ}$, latitudes between -15 and $+15^{\circ}$ ) with the same criteria as for the inner disk. The same offset between the data and models at low metallicities observed for the inner disk is seen on the bulge APOGEE data, with the number of stars overpredicted by the models at $[\mathrm{Fe} / \mathrm{H}]<-0.7$ dex.

The dip is not visible in the MDF of the APOGEE data (Fig. 6, top plot), but the overall distribution is nonetheless well reproduced by the model at $[\mathrm{Fe} / \mathrm{H}]>-0.5$ dex. A reason for the absence of the dip may be due to the distance distribution of APOGEE stars in the Galactic bulge, which is biased towards the near side of the bulge (see, e.g., Fig 2 in Ness et al. 2016), and against the lower-metallicity stars. A discussion of this distance bias and its impact on the bulge MDF is discussed in Fragkoudi et al. (2018).

In several other datasets, the bimodality of the bulge MDF is apparent. Gonzalez et al. (2015) found two components centered on $[\mathrm{Fe} / \mathrm{H}]=-0.31$ and +0.26 dex. Uttenthaler et al. (2012) found a transition at $[\mathrm{Fe} / \mathrm{H}] \sim 0$ dex (their Fig. 8), and two peaks at $[\mathrm{Fe} / \mathrm{H}]=-0.57$ and $+0.30 \mathrm{dex}$; although their field, being at $b=-10^{\circ}$, shows a much less prominent metal-rich peak. Zoccali et al. (2017) analyze 26 different fields and find a transition between two main peaks at about solar metallicity in most fields. As for the inner disk, in our model, the dip in the MDF corresponds to the lull in the star formation at $\sim 8$ Gyr marking the transition from the thick to the thin disks.

Overall, we consider the match between the model and the data to be satisfactory given that (1) the model is entirely designed by fitting chemical abundances of stars of the inner disk sequence observed at the solar vicinity, and no tuning has been introduced to fit the inner regions; (2) the uncertainties that still exist in the metallicity scales, and the apparent variability between fields at the same latitude (Zoccali et al. 2017); and (3) the difference in sampling between the four different datasets considered here.

\subsection{The bulge age-metallicity relation}

Recent evidence that the bulge also contains young stars comes from Bensby et al. (2013), who have shown that stars with age $<8$ Gyr exist in the bulge, confirming other previous findings (see, e.g., van Loon et al. 2003, and references therein). Figure 7 shows the comparison between our model AMR and the bulge data from Bensby et al. (2013). Obviously, the ages have large uncertainties, but the model is nonetheless a good representation of the data, with no obvious disagreement.

\subsection{Alpha abundances}

Because the high-alpha abundances constitute also an age sequence (Haywood et al. 2013), it is interesting to compare our model to the $[\mathrm{Fe} / \mathrm{H}]-[\alpha / \mathrm{Fe}]$ data and to the alpha element distribution.

Figure 6 (middle and lower plots) shows the alpha element distribution function (both as $[\alpha / \mathrm{Fe}]$ and $[\alpha / \mathrm{H}]$ ) of APOGEE stars compared to our models. As in Haywood et al. (2016a) on the inner disk data, the fiducial model is a good representation of the bulge $[\alpha / \mathrm{Fe}]$ distribution. The smoothed model shows less good agreement, the reason being the more prominent tail of stars at metallicities lower than $-0.7 \mathrm{dex}$, and which produces a third bump at $[\alpha / \mathrm{Fe}] \sim+0.35$. The comments made about the APOGEE MDF also apply to the $[\alpha / \mathrm{H}]$ distribution: the comparisons are satisfactory overall, with a significant overproduction of stars at low $[\alpha / \mathrm{H}]$ (old objects).

Figure 8 shows the silicon abundance as a function of $[\mathrm{Fe} / \mathrm{H}]$ and age for the microlensed stars in Bensby et al. (2013). The model does not reproduce the upturn seen in data from Bensby et al. (2013) at super-solar metallicities, but we note that the error bars are large at these metallicities, our model still being compatible with the data within one sigma, and that this upturn is not always observed. Neither Gonzalez et al. (2011, 2015), nor Ryde \& Schultheis (2015) report evidence of such a feature in any of the alpha elements they have studied. The bottom plot of Fig. 8 shows the age-[Si/Fe] distribution of Bensby et al. (2013). As for the age- $[\mathrm{Fe} / \mathrm{H}]$ distribution, the models are compatible with the data, but the data offer no real constraint because of the large uncertainties on ages.

\subsection{Conclusion}

To conclude, our fiducial model provides a good representation of a large range of observational constraints of the bulge: $\mathrm{MDF}, \alpha-\mathrm{DF},[\mathrm{Fe} / \mathrm{H}]-[\mathrm{Si} / \mathrm{Fe}]$ distribution, age- $[\mathrm{Si} / \mathrm{Fe}]$ and agemetallicity distribution with the same model as the inner disk, 


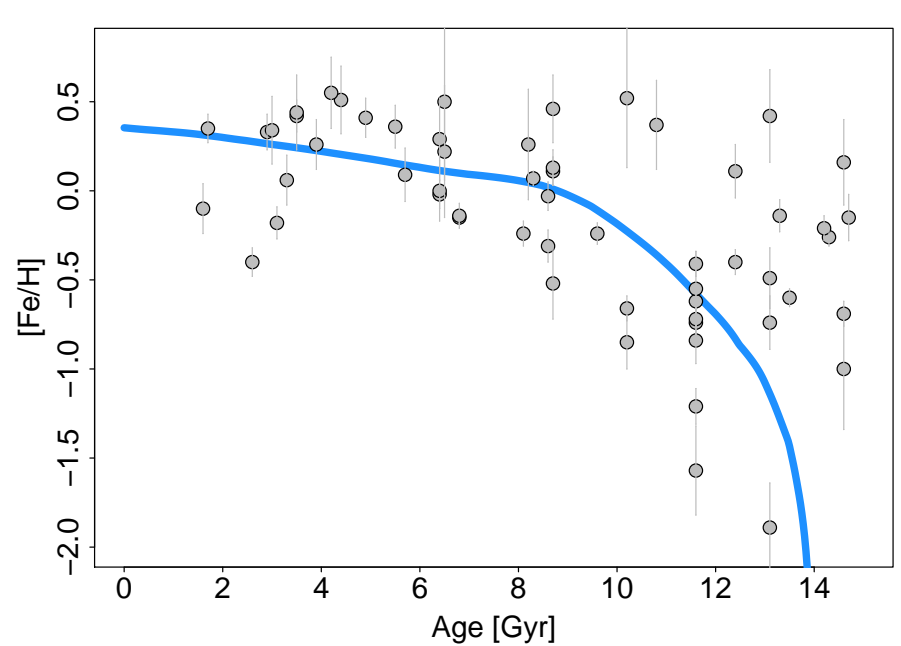

Fig. 7. Age-metallicity distribution of the bulge from Bensby et al. (2013) compared to our models.

which we find remarkable given that the model (for both the inner disk and bulge) was built only from the comparison with inner disk stars sampled at the solar vicinity. The model so far has no free parameters (apart from the initial assumption of a CBM), while in standard chemical evolution models of the bulge (e.g., Tsujimoto \& Bekki 2012; Grieco et al. 2012), the datasets are fitted allowing for several additional (and unconstrained) parameters, such as the gas accretion timescale and the IMF; these usually differ from those of the disk, but in our case the model is the same.

\section{Discussion: The inner disk-bulge population}

The application of the CBM to the bulge and inner disk is valid if these can be considered as forming unique system in all their characteristics, and if their evolution was not radially dependent. We discuss these two points in the following.

\subsection{Are the inner disk and bulge the same populations?}

If the inner disk and bulge are the same population, their kinematic, chemical, and age characteristics must be compatible. The compatibility of the kinematic properties of the disk and bulge will be discussed at length in Di Matteo et al. (in prep.) and are not discussed here. We focus here on the chemical properties and on the age problem of the bulge.

- The MDF. The possible identity of the bulge and the disk has been questioned observationally, in particular on the basis of the strongly different MDFs of the bulge and solar vicinity (see, e.g., McWilliam 2016).

In the previous section, we explained why these two should not be compared, while we have shown that there is good evidence for a similar MDF of the inner disk and bulge. This similarity appears both in the width and in the shape of the MDF, and both can be reproduced by the same chemical evolution model. It is also shown in Fragkoudi et al. (2017) that the mean metallicity maps of the bulge as a function of longitude and latitude in the model are in good agreement with the APOGEE data.

- The abundance ratios. Because the bulge is mainly a bar made of thick- and thin-disk stars formed inside the OLR, as argued in Di Matteo et al. (2014), the chemical abundance
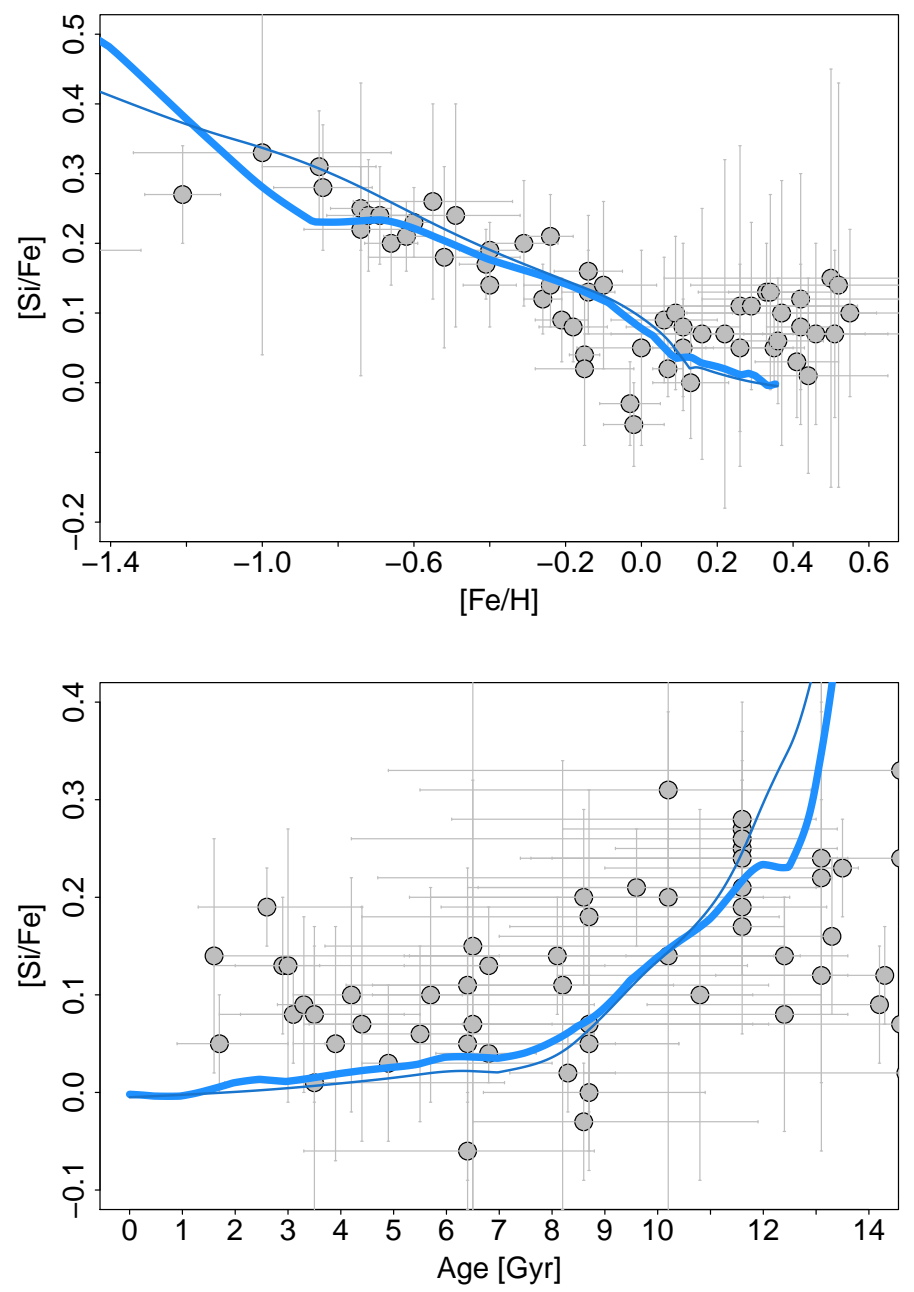

Fig. 8. $[\mathrm{Fe} / \mathrm{H}]-[\mathrm{Si} / \mathrm{Fe}]$ and age- $[\mathrm{Si} / \mathrm{Fe}]$ distributions of the bulge from Bensby et al. (2013) compared to our models (top and bottom respectively).

patterns of the bulge and the disk inside the OLR should be similar. Classically, chemical abundances of the solar vicinity and bulge are compared directly, while in fact the solar neighborhood, being a transition zone between the outer and inner disks Haywood et al. (2013), is polluted by outerdisk stars and stars formed at the OLR, which are essentially absent inside the OLR. The resulting differences, while expected, have been used to argue that the bulge and the disk have different origins. This is one of the important points to take into account when comparing the disk and bulge abundances. The other crucial point is systematic error.

To assess if the differences between the thick-disk and bulge stars are real, we must understand the level of systematic error to be expected between different samples. For instance, if the systematic errors measured on two samples of bulge stars are of the order of the differences measured between the bulge and the local thick disk, then we may infer that these differences are not significant. Both aspects are explored in detail in Appendix A. We show that when these caveats are taken into account, either the match for several element abundances is remarkable (alpha elements, $\mathrm{Ni}, \mathrm{Zn}, \mathrm{Cr}, \mathrm{Ba}, \mathrm{Eu}$, $\mathrm{La}$ ), or the differences, when they exist, are likely to remain within the level of systematic error $(\mathrm{Mg})$. Differences are not explained for some elements $(\mathrm{Cu})$, but the usually small number of existing datasets prevents any strong conclusions. 
- The age of the bulge. The supposedly exclusively old age of the bulge (Clarkson et al. 2008; Valenti et al. 2013; Nataf 2016) is cited as one of the main differences with the disk, the hurdle being the CMD of the bulge in the SWEEPS field observed by the HST (Clarkson et al. 2008). Haywood et al. (2016b) explored in detail how stars following the agemetallicity relation of our model would superpose in the CMD to give the tight turn-off that has been observed in Clarkson et al. (2008). But the main constraint comes from the wide spread in metallicity observed in the bulge: combined with an exclusively old bulge. This would automatically generate a CMD with an overly wide turn-off compared to the observation. There is presently no other way to have a CMD compatible with the observation than to assume that a significant fraction of the stars are younger than 8 Gyr. This means that the narrow turn-off of the CMD, which was cited as evidence of a coeval bulge, is in fact a demonstration of the contrary. The metal-rich stars have to be younger than $8 \mathrm{Gyr}$ to allow for the observed wide spread of metallicities to be compatible with a narrow turn-off. We note, as already mentioned in Haywood et al. (2016b), that the tight turn-off of the bulge seen in the SWEEPS field is also evidence that the age-metallicity relation in the inner disk and bulge must be tight, otherwise a significant spread in metallicity at a given age would produce a significant spread in color at the turn-off that is not observed. The most recent results from Bensby et al. (2017) confirm their previous findings (Bensby et al. 2013) and show that the fraction of stars younger than $8 \mathrm{Gyr}$ is about $50 \%$. This is higher than the percentage we estimated in the SWEEPS field (35\%, Haywood et al. 2016b), but a significant fraction of the stars in the Bensby et al. (2017) dataset is observed closer to the plane, favoring the young population Ness et al. (2014).

\subsection{Three hints that the formation of the thick disk was not inside-out}

That the whole inner disk and bulge can be described by a simple CBM as shown by the good match obtained on the MDF, alpha-metallicity distributions, age-metallicity relation and age$[\alpha / \mathrm{Fe}]$ relation, implies that no significant differentiation of the chemical evolution as a function of radius occurs within the limits of the system taken into consideration. This is at variance with the assumptions of the inside-out formation of the disk, which is commonly implemented in GCE models by parametrizing the infall timescale of gas accretion as a function of the distance to the Galactic center (see e.g., Minchev et al. 2013; Kubryk et al. 2015a; Loebman et al. 2016). The disk is formed through longtimescale infall: in Fig. 4 from Kubryk et al. (2015a) the infall timescale is between 4 and 8 Gyr at 3-7 kpc from the Galactic center; in Minchev et al. (2013) it is equal to $1.82 \mathrm{Gyr}$ at $3 \mathrm{kpc}$, and equal to $6 \mathrm{Gyr}$ at $7 \mathrm{kpc}$ ). The following points contradict the predictions of this scheme and of the inside-out formation scenario.

- The disk scale length does not increase with time. The insideout formation scenario implies that disks grow as a function of time; evidence for which is given by the increase in time of their scale length (see, e.g., Kubryk et al. 2015a, their Fig. 1). In our model, no substantial increase of the inner disk scale length is expected (Haywood et al. 2015) because the model is a closed box, and no inside-out process operates. We insist that we are discussing here the disk inside $R \lesssim 6-7 \mathrm{kpc}$, possibly corresponding to the region inside the bar OLR.
Using the SEGUE survey, Bovy et al. (2012) showed that the scale length of the inner disk remains constant with time. This is confirmed with the APOGEE survey (Bovy et al. 2016), which shows that stars that are on the inner disk sequence (high- $\alpha$ sequence in Bovy et al. 2016 paper) have remarkably constant density profiles (their Fig. 11). The most metal-rich mono-abundance group in the work of Bovy et al. (2016, Fig. 11 lower plot) has $[\mathrm{Fe} / \mathrm{H}] \sim+0.3$ dex, which is not the terminal metallicity of the solar vicinity, but the metallicity typical of the inner thin disk. Its scale length is $1.67 \mathrm{kpc}$ when fitted with a single exponential over the whole distance range from 4 to $14 \mathrm{kpc}$, but $1.25 \mathrm{kpc}$ only if fitted with a broken-exponential, which does not point to an increase in the scale length, even in the thin inner disk. The result of Bovy et al. (2016) clearly demonstrates that the scale length of the thick disk shows no increase. This is in agreement with the observation of MW-type galaxies at redshifts above 1 (when the thin disk was not yet in place) which shows also that their growth is self-similar and not inside-out (van Dokkum et al. 2013; Morishita et al. 2015).

- The radial metallicity gradient in the thick disk is flat. In the inside-out formation scenario, the gradient at the end of the thick disk phase is expected to be steep. In fact, typical predicted gradients are of the order of $-0.125 \mathrm{dex} / \mathrm{kpc}$ (see Fig. 4 in Kubryk et al. 2015a, Fig. 2 in Minchev et al. 2013). In contrast, we considered (Haywood et al. 2013) that the tight age-metallicity relation in the thick disk implies that the ISM during formation of the thick disk is essentially well mixed, due to the high turbulence that existed at this epoch (Elmegreen \& Elmegreen 2006; Förster Schreiber et al. 2006). These are also the conditions of our model, where the ISM is assumed to be well mixed. This is supported by the analysis of the SEGUE survey by Cheng et al. (2012), who find that essentially no radial gradient is visible on thick-disk stars, as defined by stars between 1 and $1.5 \mathrm{kpc}$ from the Galactic plane. The lack of a gradient in the thick disk is not due to the effect of radial mixing. We emphasized in Haywood et al. (2013) that mixing (by either churning or blurring) of an existing gradient would generate a dispersion in the AMR, which is not observed. If the disk had uniform chemical characteristics independent of radius, at the end of the thick disk formation, it is possible that the subsequent formation of the thin disk may have led also to a flat metallicity distribution in this population. We emphasize that existing measurements (e.g., Hayden et al. 2015; Bovy et al. 2016) find a steep radial gradient in the thin disk but are restricted to within $\pm 2 \mathrm{kpc}$ from the Sun radius, essentially excluding the distance range considered here $(R \lesssim 6 \mathrm{kpc})$. In fact, Andrievsky et al. (2016) suggest that the gradient may be flat within $R<6 \mathrm{kpc}$ in the thin disk.

- Chemical abundances have a very small dispersion at a given age in the thick disk. In the inside-out scenario, at early times, the SFR is more intense in the inner parts of the disk, producing a negative radial gradient of alpha abundances (alpha abundances decreasing with increasing radius) and metallicities (chemical evolution being faster in the inner part, the metallicity is higher in the inner disk at a given age). Because these stars are now on significantly eccentric orbits, a spread of alpha abundances would be expected for stars of any given age at all radii. A significant dispersion is also predicted by these models in metallicity and $[\alpha / \mathrm{Fe}]$ as a function of age, and the same is expected in the $[\mathrm{Fe} / \mathrm{H}]-[\alpha / \mathrm{Fe}]$ plane. Figure 9 illustrates the chemical tracks that may be 

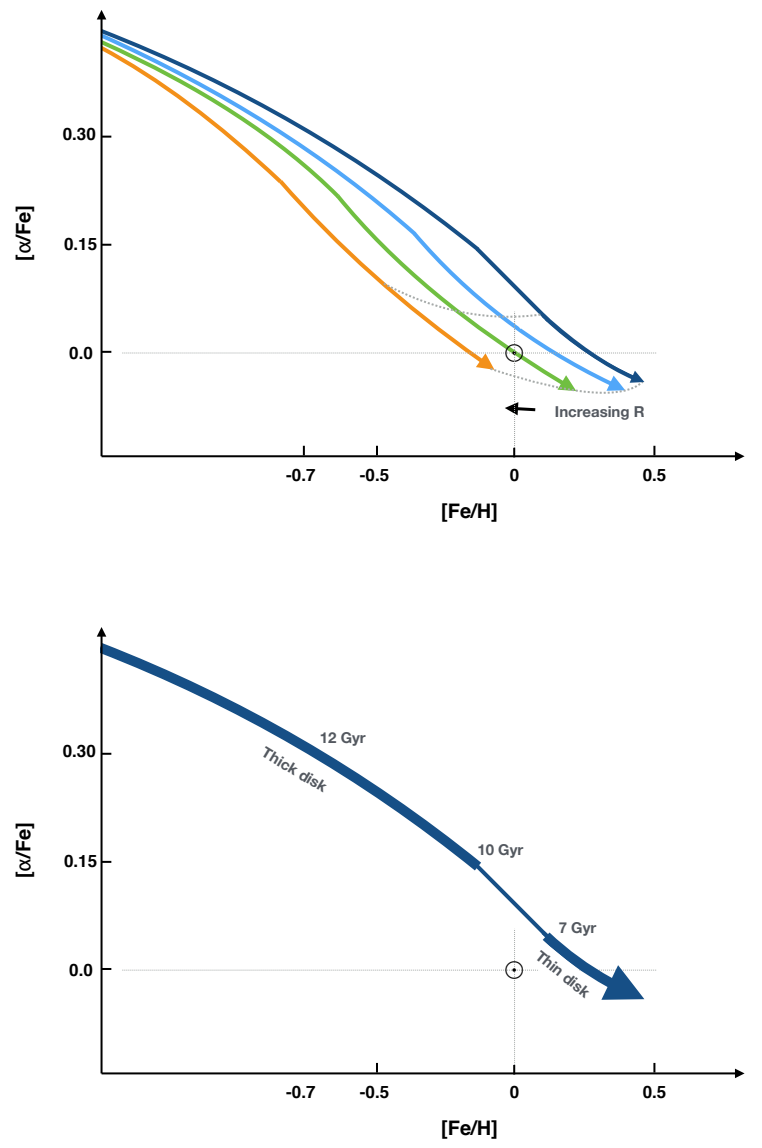

Fig. 9. Two different illustrative sketches of the inner disk $[\mathrm{Fe} / \mathrm{H}]-[\alpha / \mathrm{Fe}]$ distribution: in case of the inside-out scenario (top), and for the scenario presented here (bottom). We emphasize that these schemas are meant to represent the evolution of the inner disk only (not the whole disk). The position of the Sun (which, in the second scenario, does not belong to the inner disk evolution) is represented on each plot, together with indicative ages along the chemical track in the bottom plot. On this plot, the thinner segment (between 10 and $7 \mathrm{Gyr}$ ) corresponds to the quenching episode of star formation found in Haywood et al. (2016a). In the first scenario, a spread in metallicity and alpha abundance is expected at a given age in the inner disk.

expected in this plane: in the case of the inside-out formation of the thick disk (plot a), the inner thick disk reaches higher metallicities at a given $[\alpha / \mathrm{Fe}]$, while due to a slower evolution, the outer thick disk would reach the same metallicities at later times. In contrast, if the thick disk forms from an ISM whose homogeneity is maintained by high turbulence, its chemical evolution will describe a unique track. The small dispersion in chemical characteristics at the end of the thick disk phase is exemplified by the age- $[\alpha / \mathrm{Fe}]$ relation. Even taking into account the observational uncertainties on age and alpha abundances, the observed spread is significantly smaller than predicted by these models; see Haywood et al. 2015.

These three pieces of observational evidence strongly suggest that the thick disk formation was not inside-out, and that the inner disk and bulge can be suitably represented by a model where no spatial differentiation is introduced. They also imply that any detection of radial migration among stars within the OLR (the region where radial migration is expected to be the strongest) based on chemical characteristics would be difficult.

\section{The significance of the closed-box model and the history of gas accretion in the Milky Way}

The model described in the previous sections, in spite of its simplicity, provides a fair representation of a surprisingly large range of observational constraints of the inner disk and bulge. The aim of the present section is to try to understand why. Standard chemical evolution models follow two implicit principles: the SFH follows the accretion history, and for this to be effective, the SFR is proportional to the gas density at all times. None of these are applicable to our CBM, and we must understand what this implies for the accretion history and for the SFR in the MW.

The latest paradigm of how gas is accreted on galaxies (see Sect. 6.1) through cold flows, suggests that most of the gas may have been accreted rapidly $(z>2-3$, or in the first $2-3$ Gyr) in the central parts of galaxies. The CBM is different from this picture, because it describes an instantaneous accretion, where most accretion more likely occurred at $z>6$. This may be the reason for the discrepancy observed at $[\mathrm{Fe} / \mathrm{H}]<-0.5$ $\operatorname{dex}(z>3$ in the model). The model satisfactorily represents the data above $[\mathrm{Fe} / \mathrm{H}]=-0.5$ though, at epochs when accretion may still have been quite substantial (down to $z \sim 2$, corresponding to $[\mathrm{Fe} / \mathrm{H}] \sim 0$ dex in the model). This means either that in the case of the Milky Way, most accretion was terminated at significantly earlier times (at $z>3$ ), or that the building of the disk between $z=2$ and $z=3$ was not dependent on the accretion rate. This is discussed in Sect. 6.1.1.

The second constraint that limits the CBM is that any MW model with high gas content, and in particular the CBM, is bound to produce an unrealistic age distribution if the SFR is directly dependent on the total amount of gas. Yet, to be valid, the model also has to be on the SK relation. We explore in Sect. 6.2 the consequences on the gas content of the model if this is to be verified. Also, a strict dependence on the SK law ignores possible variations in the star-formation efficiency (e.g., Schreiber et al. 2016). The data for the MW, for instance, show evidence for a quenching episode occurring while the total amount of gas and its surface density is thought to have been quite high (Haywood et al. 2016a), if MW evolution was similar to other galaxies of the same stellar mass. This result implies that the star formation efficiency must have varied significantly at this epoch. Such a variation in the SFE cannot be explained in standard GCE models. Standard GCE models enforce a strict relation between gas density and the SFR, and the shutdown in the SFR that occurred 10 Gyr ago would only be modeled by a decrease in the accretion rate and a decrease in the gas density. A variation of the star formation efficiency implies that the accretion rate and a direct relation, through the SK law for example, cannot be the sole or even the most significant factor determining the SFH. Other factors must regulate the SFR via the starformation efficiency, such as mechanisms to enhance turbulence, like a bar, which we invoked to explain the quenching episode of the MW (Haywood et al. 2016a), stellar feedback (e.g., Dib 2011), or AGN feedback, which may quench or enhance star formation, depending on the distance to the black hole (e.g., Robichaud et al. 2017).

\subsection{The accretion history of our Galaxy}

\subsubsection{Accretion at $z>2$}

By the early-00s, modelers had sufficient computing power to calculate both the dynamical properties of dark matter over cosmological scales in a hierarchical Universe and the evolution of gas in galaxies regulated by simple heating and cooling 
prescriptions. From their simulations, they found that not all, and perhaps not even most of the accreting gas depending on halo mass would be heated to high temperatures when passing through the halo accretion shock, but would instead penetrate down into the halo as filaments of gas and dark matter (Birnboim \& Dekel 2003; Kereš et al. 2005, 2009; Ocvirk et al. 2008; Agertz et al. 2009). Within this framework, galaxies with the halo mass of the MW acquire most of their gas early $(z \gtrsim 2)$ in their evolution and largely through the cold mode of accretion (Dekel \& Birnboim 2006; Woods et al. 2014; Tillson et al 2015), a fundamental requirement to form a massive thick disk of age $>10 \mathrm{Gyr}$.

Describing an "instantaneous accretion", the CBM differs from this picture and if the SFR was directly dependent on gas inflow, continuous accretion to $z \sim 2$ would produce a model different from the CBM. As mentioned above, if the deficit in the number of stars seen at $[\mathrm{Fe} / \mathrm{H}]<-0.5$ dex compared to the $\mathrm{CBM}$ is confirmed, it may correspond to a "G-dwarf" problem moved to $z>3$, possibly testifying to a mismatch between the CBM and substantially longer-lasting gas accretion. At metallicities above $[\mathrm{Fe} / \mathrm{H}] \sim-0.5 \mathrm{dex}$, the model is a satisfactory match to the data, and it is interesting to try to understand why. The first possible answer is that accretion was fast enough (essentially terminated within $z>3$ ) for the CBM to be a good approximation starting from $z \sim 3$. The second possible answer is that the building of the disk - its SFR - was not directly dependent on gas accretion, as we now speculate.

Several studies have also shown that in the phase where the cosmic SFR density increases, $z \gtrsim 2$, the volumetric gas accretion rate exceeds the SFR of the ensemble of galaxies, resulting in a general "gas accumulation phase" (Davé et al. 2012) or "gas accretion epoch" (Papovich et al. 2011). During this epoch, the reservoir of gas is filled up due to the difference in the accretion and SFRs. Observation shows that MW-mass galaxies at redshift $z \sim 1.3$ have molecular gas fractions of $\sim 40-50 \%$ (Tacconi et al. 2013; Saintonge et al. 2013; Dessauges-Zavadsky et al. 2015; Papovich et al. 2016). This implies that MW-mass galaxies have already acquired all the gas necessary to make present MW-type galaxies and this is without considering the substantial impact of mass return (Leitner 2012); see Sect. 6.1.3.

Perhaps more importantly, several studies have argued that once galaxies approach the peak of their star formation at $z \sim 2-3$, they adopt a quasi steady state balance between star formation, gas accretion, and outflows (Bouché et al. 2010; Dutton et al. 2010; Davé et al. 2012; Krumholz \& Dekel 2012; Lilly et al. 2013) and the gas mass remains approximately constant (Davé et al. 2012). Numerical simulations seem to show a similar phenomenon (Stinson et al. 2015). What these models express is that at these epochs, the SFR is not simply proportional to the gas supply (Lehnert et al. 2015), and some mechanism breaks a direct coupling between star formation and the gas accretion rates (e.g., Peirani et al. 2012; Lehnert et al. 2015). Feedback is offered as the mostly likely plausible solution, as it can limit the SFR to values compatible to the observed one (Lehnert et al. 2015; Agertz \& Kravtsov 2015, their Fig. 4) and in fact, the MW likely drove significant outflows in its early evolution (Lehnert et al. 2014). Moreover, outflows and largescale gas recycling (fountains) provide an explanation for the elemental abundances of the outer disk and its connection with the metallicity of the inner disks (Haywood et al. 2013). Simulations by Perret et al. (2014) suggest that stellar feedback and accretion might provide a mechanism to saturate SF in highredshift galaxies by maintaining a high level of turbulence and fragmentation in the gas. In disks already saturated with gas, the variations of its supply may not be the driving factor for the level of the SFR and its variations, as suggested previously. Hence it is possible that the CBM described here provides a good representation of the data in the range $2<z<3$ because it captures two fundamental features of accretion of gas through cold flows: (1) concentrating huge amount of gas rapidly in the central part of disks and (2) lifting the direct dependance of SFR on gas accretion rate. Finally, if gas accretion continues to $z \sim 2$, our description is valid if its impact on the gas out of which stars are forming in the inner $10 \mathrm{kpc}$ of the Galaxy is limited. At these epochs, according to van de Voort et al. (2011), cold and hot gas accretion brings similar amount of gas into halos. The accretion onto galaxies themselves is lower by roughly a factor of three to four. The cooling time of the hot halo gas is long and thus the accretion rate is perhaps low (e.g., Maller \& Bullock 2004). The cold accreted gas will have higher angular momentum with decreasing redshift and will be preferentially accreted onto the outer parts of the disk (e.g., Danovich et al. 2015). Such gas may move into the inner disk due to, for example, tidal torques from the inner disk but over a longer timescale than its accretion time scale. Moveover, the rate of cold gas accretion onto galaxies will likely be mitigated by feedback from stars in the galaxy itself (e.g., Dubois et al. 2013; van de Voort et al. 2016). Interestingly, star formation intensity in the MW at $z \gtrsim 2$ was strong enough to generate significant outflows (Lehnert et al. 2014). Therefore, a complex interplay between inflows and outflows generated by feedback could lead to an equilibrium between gas supply and removal. So while qualitatively there are reasons to a priori suspect that gas accretion had a limited impact on the effect of chemical evolution of the inner $10 \mathrm{kpc}$ of the disk, to access this possibility quantitatively will require a more detailed model and simulation.

\subsubsection{The quenching episode and accretion after $z \sim 2$}

Simulations suggest that for MW mass halos, the gas accretion rate peaks at redshifts of $z \sim 2-3$ and then declines fairly steeply thereafter (e.g., van de Voort et al. 2011; Woods et al. 2014). It is after the peak that the MW started to quench its star formation (Fig. 1; Haywood et al. 2016a). While the decline in the gas accretion rate coincides with the quenching epoch in the evolution of the MW, representing also the change from thick to thin disk formation, the decline itself is, strictly speaking, probably not responsible for either the quenching or the change from thick to thin disk formation. In our model, there is still plenty of gas in the MW disk during this epoch, of the order of $40-50 \%$ of the total mass as is typically observed on MW-type galaxies at $z \sim 1.5$ (e.g., Daddi et al. 2010; Genzel et al. 2015). If star formation proceeded with an efficiency typical of disk galaxies at high redshifts, it would have proceeded unabated. Therefore some other process(es) must have triggered this quenching phase, which is (are) not directly related to the decline in the gas accretion rate at $z \lesssim 2$. Haywood et al. (2016a) proposed that the bar, by sustaining significant turbulence in the gaseous disk, must have prevented SF from occurring at a typical efficiency. Khoperskov et al. (2018) show, using N-body hydrodynamic simulations, that it is possible for the bar to suppress the star formation efficiency. It is most probable, however, that the birth of the bar was made possible by the disk settling due to the decrease of the gas fraction in the disk, passing from turbulent disk to a marginally unstable disk (Ceverino et al. 2017; Hayward \& Hopkins 2017; Ma et al. 2017). The decrease in the gas fraction, is, in turn, probably related to the decrease of the gas accretion rate. That means that although the end of the cold 
accretion flows is not directly responsible for the quenching, the two could be causally, but only indirectly related. This also would explain why these events, that is, the decrease in the gas accretion rate, the formation of a bar, and quenching, all seem to occur at approximately the same epoch.

The thick and thin disks, although separated in time by about 1.5-2 Gyr, show continuous chemical properties, and in particular a continuous level of alpha element abundance (in the sense that the thin disk starts at the same $[\alpha / \mathrm{Fe}]$ where the thick disk ends; Fig. 3). For the gas accretion rate to be in equilibrium with SFR of the post-quenched thin disk requires a gas accretion rate of $\sim 3 M_{\odot} \mathrm{yr}^{-1}$ (which is also the approximate rate in simulations, Woods et al. 2014). Assuming that the amount of molecular gas in the disk at the end of the thick disk formation $(z \sim 1.5)$ was $40 \%$ of the total baryonic mass of the disk (which we take as $5.10^{10} M_{\odot}$ ) and the metallicity of the ISM at this epoch was about solar, if the Galaxy continued to accrete gas at a rate of $3 M_{\odot} \mathrm{yr}^{-1}$ during the long quenching episode lasting $\sim 2 \mathrm{Gyr}$, then $\sim 0.6 \times 10^{10} M_{\odot}$ of gas would have been added to the ISM. If the metallicity of this newly accreted gas was $[\mathrm{Fe} / \mathrm{H}]=-1 \mathrm{dex}$, then the ISM of the Galaxy at the beginning of the thin disk formation would have been 0.1 dex less metal-rich than the ISM at the end of the thick disk phase (a gas at a metallicity of -2 dex would give a similar value). If the accreted gas was devoid of alpha elements, the age-alpha relation of Fig. 3 would show a hiatus or an offset of $+0.1 \mathrm{dex}$ in $[\alpha / \mathrm{Fe}]$ at $\sim 7 \mathrm{Gyr}$ compared to the data. Higher amounts of alpha elements would only increase the offset. A hiatus of this magnitude is not observed. The measured dispersion in observed $[\alpha / \mathrm{Fe}]$ at ages of less than $8 \mathrm{Gyr}$ (excluding the $3 \sigma$ outliers) is only $\sim 0.02 \mathrm{dex}$, and even this is an upper limit because it is consistent with being entirely due to observational uncertainties. In practice, with these assumptions, to keep variations in the $[\alpha / \mathrm{Fe}]$ ratio below $1-\sigma$ dispersion, any accretion must have had a rate $<0.6 M_{\odot} \mathrm{yr}^{-1}$. This effectively means that gas accretion in the inner disk had come to an end.

\subsubsection{The case for negligible accretion in the MW inner disk $(R<7 \mathrm{kpc})$ since $z=1$}

Models showing galaxies that form inside-out have shorter infall timescales in the inner regions compared to the outer regions, and so an age distribution weighted towards older stars in their inner disks. Because models parametrize the SFR by SchmidtKennicutt type laws, the resulting SFHs follow the accretion history which are a steep function of time and thus produce a negligible amount of young stars in the inner regions. In Minchev et al. (2014), for example, the disk at 3-5 kpc is overwhelmingly dominated by old ( $>6$ Gyr) stars. Since the SFH follows the accretion history, these models are unable to produce sufficient numbers of "young" (age $<8 \mathrm{Gyr}$ ) and hence metalrich $([\mathrm{Fe} / \mathrm{H}]>0$ dex $)$ stars to reproduce the peak visible in the data at $[\mathrm{Fe} / \mathrm{H}] \sim+0.25$ dex. Similarly, in standard models of the bulge, where this component is represented by exclusively old populations because of the assumed SK law (which we depart from) and a short gas accretion timescale induce the very fast conversion of gas to stars.

At larger radii in these types of models the accretion timescale is assumed to be long, and thus most of the stellar mass is formed after $z=1.0$. This is discussed for example, in Fraternali (2013), and it is interesting to compare their scheme with ours. Fraternali (2013) states that "most of the star formation and therefore most cold accretion must take place during the hot-mode phase" (or at $z<1-2$ ), which is clearly at odds with our findings. Assuming that the thick disk is part of their description, only about $25 \%$ of the stellar mass of the disk is formed at $z=1.0$, which is probably incompatible with our SFH and the massive thick disk we find. In addition, while in their scheme they describe the progressive shift of the accretion towards the outer disk, which we also expect, in the inner parts $(R<6 \mathrm{kpc})$ most of the gas is accreted at times $<8 \mathrm{Gyr}$, and only in the last $2 \mathrm{Gyr}$ does the accretion in the outer parts start to dominate. In contrast, we find that to describe the abundances of the inner disk and bulge, prolonged infall is not necessary. Hence, we do not find the contradiction discussed by Fraternali (2013) between a cold gas accretion which supposedly dominated the accretion history at $z \gtrsim 1-2$ and a significant fraction of the stellar mass that would have formed mainly after $z=1$ in their picture.

At the end of the quenching phase, our model implies that the gas fraction in the Galaxy was still high, which, coupled to a low (2-3 $M_{\odot} \mathrm{yr}^{-1}$ ) SFR implies an SFE significantly lower than at the present time. For an SFR of $2-3 M_{\odot} \mathrm{yr}^{-1}$, a gas fraction of $30-40 \%$ (or $1.5-2 \times 10^{10} M_{\odot}$ ) would yield a depletion timescale of 5-10 Gyr, which, for a disk 7 Gyr old, suggests that the mass of gas at the end of the quenching episode was sufficient to maintain relatively weak but significant star formation up to the present time (even if all the gas counted in the model may not have been available to form stars; see below). Hence, we would suggest that prolonged substantial gas accretion is not necessary to explain the properties of the stellar disk found inside the solar orbit, which represents most of the mass of the MW. These results also suggest that the present lack of observed evidence for substantial accretion, being a factor of 10 below the actual SFR of the MW, is expected.

We emphasize that our description refers to the inner disk of our Galaxy, and it does not preclude that a substantial amount of gas may still be accreted in the outer parts of the MW in a way described by gas accretion models at late times. Late-time accretion would have a relatively high angular momentum and could result in the extended HI disks observed in the MW and other MW-like galaxies (as discussed for example in Lehnert et al. 2015). Other mechanisms such as gas accretion driven by energy injection into the halo by disk star formation ("galactic fountain" models) might also be viable mechanisms for sustaining disk star formation (Armillotta et al. 2016).

\subsection{Placing our model on the Schmidt-Kennicutt law}

We now study how our model is related to the Schmidt-Kennicutt (SK) relation, keeping in mind that we have a measure of the relative SFH (Fig. 1). We deduce ${ }^{2}$ the absolute SFR by fixing the total current stellar mass of the Galaxy at $5 \times 10^{10} M_{\odot}$. Our model only considers gas lost during the evolution of the stellar populations in the MW (Snaith et al. 2015). This implies that after $\sim 14$ Gyr of evolution, $28 \%$ of the initial total mass of the system is in gas. To be consistent with the final mass of the MW implies that the initial baryonic mass (which is the pool in which the nucleosynthetic products ejected from stars are recycled) would be $6.4 \times 10^{10} M_{\odot}$.

A baryonic census of the MW is difficult; we must consider all the phases. However, for a CBM to be appropriate, it must at

\footnotetext{
2 As noted in this paper, our SFH describes the mass growth of the thick disk and inner thin disk. Hence, when counting the total stellar mass, we are assuming that the thin outer disk had a negligible contribution to this mass growth and to the SFH as discussed in this section. Assuming a thin disk with a scale length of $3 \mathrm{kpc}$ and a limit to the inner thin disk at $7 \mathrm{kpc}$, a very rough estimate suggests that we are missing $10 \%$ of the thin disk.
} 
least be plausible with what is currently known about the distribution of baryons in the MW itself. Although very uncertain, the distribution of mass within the MW is roughly $2.5 \times 10^{9} M_{\odot}$ in $\mathrm{H}_{2}, 8 \times 10^{9} M_{\odot}$ in HI, and $2 \times 10^{9} M_{\odot}$ in HII (Kalberla \& Kerp 2009 , and references therein). If we naively use these numbers to estimate a gas fraction of the MW, we find a total mass of $6.2 \times 10^{10} M_{\odot}$. There is of course a hot extended halo whose mass is not well determined. It could range from insignificant to the dominant gaseous component in the MW $\left(0.2-12 \times 10^{9} M_{\odot}\right.$, Miller \& Bregman 2013). In any event, we conclude that our CBM is consistent with what is currently known about the gas content of the MW.

We cannot directly equate the gas in the system that could form stars using the SK relation. It is already known that for CBMs, the application of a SK law gives unrealistic age distribution that peaks very early (e.g., Fraternali \& Tomassetti 2012) because of the huge gas surface density at early times. This is the reason why we did not use the SK law in the first place to model the SFH of the MW. At early times, our estimated SFR is $\sim 12-15 M_{\odot} \mathrm{yr}^{-1}$. If SK-type law were to hold, this would imply that either not much of the gas is able to form stars at any given time or that the star formation efficiency (SFE = SFR/gas mass) of the MW at those early times was very low. The depletion time, $t_{\text {dep }}=1 / \mathrm{SFE}$, of our model, assuming all gas can form stars, is about 4-5 Gyr at early times older than about 9 Gyr, then rises to $\sim 40$ Gyr during the quenching episode, and then is of the order of 10 Gyr until today (Fig. 10). Observations of high-redshift $(z>1)$ galaxies suggest that the gas depletion timescales are $\lesssim 1 \mathrm{Gyr}$ (Tacconi et al. 2013, 2018; Genzel et al. 2015; Schinnerer et al. 2016). Locally, gas depletion timescales are much longer: about 1-3 Gyr (Leroy et al. 2008, 2013; Bigiel et al. 2014). These gas depletion timescales are defined relative to the $\mathrm{H}_{2}$ content of the galaxies and not the total gas content as we have done.

In our model, we also assume that not all the gas that makes the reservoir in which metals are diluted is molecular, and accept that other modes of the gas, such as the hot halo, or HII regions, also take part in efficient mixing. Hence, we need to rescale our estimates of the gas depletion timescale to account for this difference to reconcile them with the observations of the galaxy population. This can be done in two ways. First using the final gas depletion time of our model and scale it to an estimate of the gas depletion time of local star-forming galaxies. If we scale our average gas depletion time during the thin disk formation phase to 2.35 Gyr (Bigiel et al. 2011), we must decrease the depletion time history of our model by about a factor of 4-5 (Fig. 10). Second, by taking into account only the fraction of gas that participates in the star formation, or the $\mathrm{H}_{2}$ relative to the HI and HII mass estimates given above, we get approximately one quarter. We do not know how this fraction of the total gas evolved with time because of the difficulty in measuring $\mathrm{HI}$ masses at high redshifts. Initial assessments show that within $z \sim 0.2$, this fraction is similar to what is observed on local galaxies (Cortese et al. 2017). At higher redshifts, using absorptions lines as a proxy for estimating the HI content of galaxies suggest an increase with increasing redshifts (Noterdaeme et al. 2012). Cosmological simulations show that this fraction should increase with redshift (see, e.g., Obreschkow \& Rawlings 2009; Lagos et al. 2011; Popping et al. 2014; Davé et al. 2017) and also show that the $\mathrm{HI}$ and $\mathrm{H}_{2}$ content may grow in approximate lock-step (Davé et al. 2017). At high redshifts, $z \gtrsim 2$, during the thick disk formation phase, using this scaling, the estimated gas depletion timescale is $\sim 1.0 \mathrm{Gyr}$, or just below. This is in reasonable agreement with gas depletion time estimates over
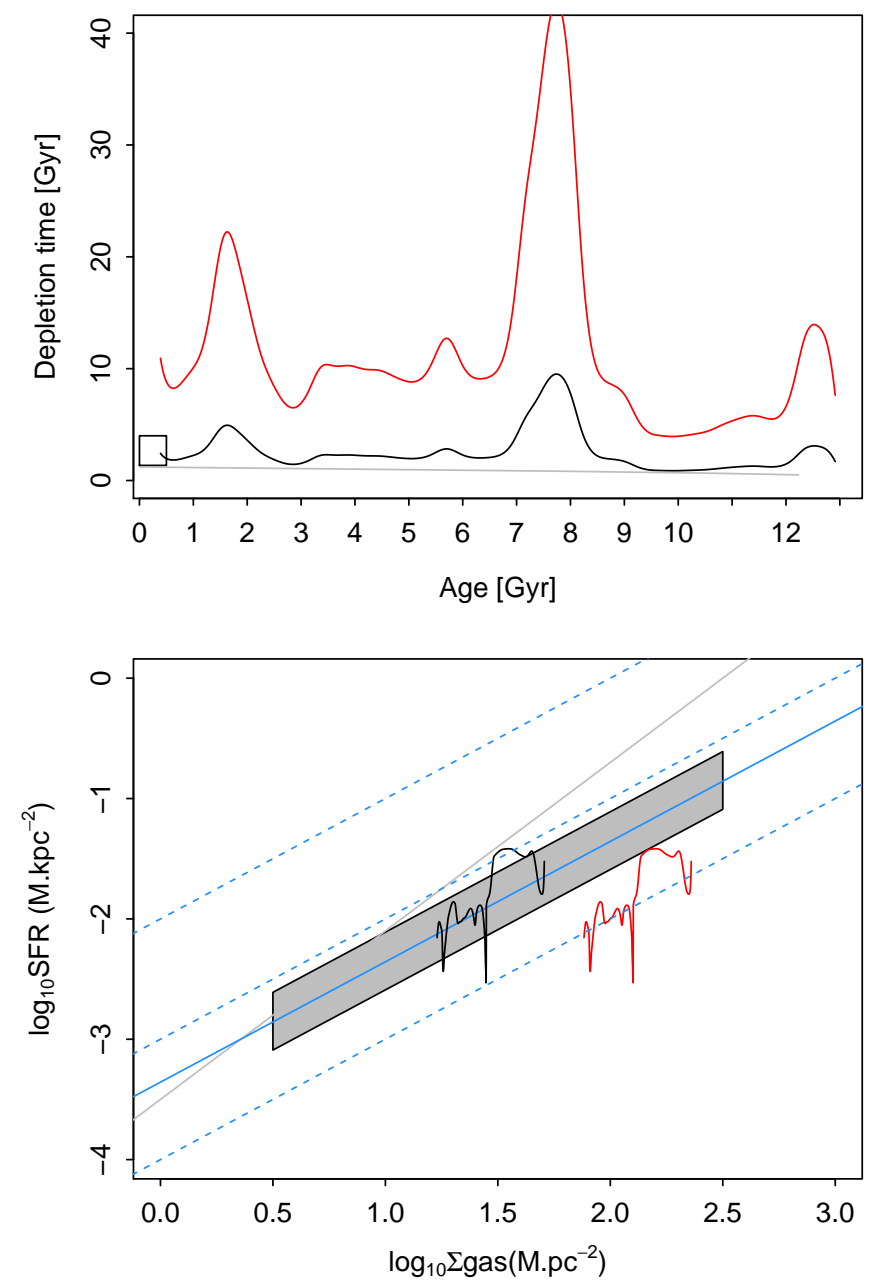

Fig. 10. Top: red curve shows the depletion timescale of the model, assuming all the gas in the system can form stars. The black curve is the depletion timescale of the model, rescaled to have a depletion timescale at present times compatible with the value of Bigiel et al. (2011) of $2.35 \mathrm{Gyr}$ (square at age $=0$ ). This implies that at any given time, 2/9 of the gas is molecular. The peak at $7.5 \mathrm{Gyr}$ corresponds to the quenching episode. The gray line is the depletion timescale of molecular gas as a function of time from Tacconi et al. (2018). Bottom: our model on the Schmidt-Kennicutt diagram, for the two cases as above. The blue line corresponds to the relation of Bigiel et al. (2011), and corresponds to a SK law with exponent $n=1$. The gray area corresponds to the 1sigma observations of Bigiel et al. (2011) for $\mathrm{H}_{2}$. The gray line is the Kennicutt law with an exponent 1.4.

those redshifts; see the estimates from Tacconi et al. (2018) on Fig. 10.

We can also use the total SFR and gas masses to determine the evolution of the MW in the gas and SFR surface density plane, the Schmidt-Kennicutt relation. By scaling the SFR and gas mass over the size of the inner disk during the formation of the thin disk as we did for the gas depletion time scaling, we can estimate the surface densities of both. Once this zero point is set, we can make a relationship between the surface densities which then simply follows the evolution of the SFR and gas mass (Fig. 10). What we find is that the MW evolves along the local SK relation (Bigiel et al. 2014) if we apply the same gas fraction scaling as we did for the gas depletion timescale. The MW in its quenching phase, the lowest point on the model curve, appears as offset from the mean 
relation, at almost $3 \sigma$, but within the observed dispersion (Bigiel et al. 2014, their Fig. 1).

The mode of star formation does not seem to cause significant deviation from the SK or gas depletion times as long as approximately a quarter of the gas is available for forming stars. At early times, the gas is highly turbulent and the intense star formation observed in distant galaxies appears to be marginally unstable (e.g., Lehnert et al. 2013). After the quenching episode, the disk settled, and simply grew as a normal spiral does with a gas depletion timescale of a few billion years. During all phases of evolution, star formation in the MW was inefficient (see Papovich et al. 2016, for a discussion of this point). MWmass galaxies represent the evolution of $\sim M_{\star}$ galaxies over a wide range of redshifts (Ilbert et al. 2013). In fact, MW-like spiral galaxies in the local universe appear to have very similar SFHs (González Delgado et al. 2017).

We conclude that the Schmidt-Kennicutt relation is an adequate description of the star formation in the history of the MW as long as most of the gas at any one time does not participate in star formation. Because of the similarities of the MW with other spirals of the same mass and because MW-mass galaxies are approximately $M_{\star}$ over a wide range of redshifts, this also must be true for the population of galaxies where most of the stars lie.

\section{Conclusions}

We have shown that the bulge and the inner disk (the disk inside the OLR) can be described by the same chemical evolution, using a CBM. The model has been compared and is compatible with the MDF, $[\alpha / \mathrm{Fe}]-\mathrm{DF},[\alpha / \mathrm{H}]-\mathrm{DF}$, age-metallicity relations, as sampled from the APOGEE catalog from $R \sim 6 \mathrm{kpc}$ to the Galactic bulge, microlensing data, and inner disk stars sampled at the solar vicinity. We summarize our main results:

When identifying the bulge with the disk, only the disk inside the OLR (which we call the inner disk) should be taken into account, because it is only inside the OLR that stars can loose sufficient angular momentum to become trapped in the bar (Di Matteo et al. 2014; Hallé et al. 2015). Hence, stars at or beyond the OLR are not expected to participate in significant number to building the inner regions (Hallé et al. 2015). It is therefore expected that the MDF of the bulge and that of the solar vicinity, which appears to be in the OLR region, are different, contrary to what is sometimes assumed (e.g., McWilliam 2016).

The chemical properties of the bulge and inner disk - MDF, age-metallicity relation, and chemical patterns of alpha abundances - are well described by the same model, which, combined with dynamical arguments, implies that the bulge is dominated by the thick disk and the thin disk inside the OLR. There is no need for an independent chemical evolution requiring particular parameters to describe most of the bulge. The model shows an excess of stars at $[\mathrm{Fe} / \mathrm{H}]<-0.5 \mathrm{dex}$, corresponding to ages greater than 11-12 Gyr, when compared to the APOGEE inner disk and bulge MDF. We suggest that this possibly gives the limit below which the CBM approximation becomes invalid.

The bimodality observed in the MDF of both the inner disk (after residual contamination by the outer disk stars is removed) and bulge, and the dip in between them stems from the quenching episode that occurred in the MW about 9 Gyr ago (Haywood et al. 2016a) and which separates the thin and thick disks. The dip in the MDF at $[\mathrm{Fe} / \mathrm{H}] \sim 0$ dex is the signpost for a common evolution of the bulge and the inner disk.
We infer that the age-metallicity relation in the inner disk and bulge must be much tighter than what is measured in the solar vicinity. The bimodality, or, more exactly, the lull in the MDF at $[\mathrm{Fe} / \mathrm{H}]=0$ dex is evidence that the AMR in the inner disk must be tight, otherwise, if the dispersion in metallicity at a given age was significant, we would not see the lull.

Using our SFH, which is determined by fitting the abundance trends with age, allows us to not assume an accretion law, and to lessen the strict coupling which is commonly assumed between the accretion law and the SFR through some Schmidt-Kennicutt star-formation prescription. Furthermore, this approach allows us to infer a sudden decrease of the star formation efficiency which must have occurred during the quenching phase $(z=1-2)$, allowing the inner disk to have sustained the same level of SF until the present time without significant replenishment. As we argued in Haywood et al. (2016a) and further demonstrate in Khoperskov et al. (2018), the formation of the bar permits the decrease of the star formation efficiency, and therefore the quenching episode.

The chemical continuity between the thick disk and thin inner disk indicates that the accretion of cold gas cannot have lasted long after the epoch of the beginning of quenching. The continuity in the chemical abundances, and in particular the $[\alpha / \mathrm{Fe}]$ ratio at the time of quenching imposes strong upper limits on the possible accretion at this epoch and tells us that accretion in the inner disk must have ended at this epoch.

The occurrence of these three events, that is, the decrease of the accretion rate, growth of the bar, and the quenching episode, at approximately the same time is not a coincidence: they are causally related. It is the quenching of the MW star formation activity which allowed the thick phase formation of the Galaxy to end and the thin disk, or secular phase, to begin. The MW provides a clear example of where morphological transformation is associated with a quenching phase.

The constraints that we obtain suggest that the gas accretion history of the MW is not the only - and sometimes not even the main - parameter determining the variations in the SFH. The establishment of the bar must have induced a significant increase in the depletion timescale (or decrease in the star formation efficiency) and permitted the inner disk to continue forming stars with no substantial replenishment until the present time. It is therefore possible that during most of its evolution, the inner disk was overabundant in gas, with the SFR at $z>2$ being limited by feedback and turbulence, and at $z<1$ by a weak star formation efficiency, making the SFH essentially independent of the history of gas accretion.

The solar vicinity is just outside the limits of the system described in this paper, being in the region where the OLR is currently located $(7-10 \mathrm{kpc})$. We discuss, in a forthcoming paper, how much the evolution of the solar vicinity remains close to the model described here, and quantify the amount of inflow necessary to explain its chemical characteristics.

Acknowledgements. The Agence Nationale de la Recherche (ANR) is acknowledged for its financial support through the MOD4Gaia project (ANR-15-CE310007, PI: P. Di Matteo), also providing the postdoctoral grant for Sergey Khoperskov. Francesca Fragkoudi was supported by a postdoctoral grant from the Centre National d'Etudes Spatiales (CNES). MH and PDM acknowledge the hospitality of Valerie de Lapparent and the "Galaxies" team at the Institut d'Astrophysique de Paris, where this work was partially developed. Funding for the Sloan Digital Sky Survey IV has been provided by the Alfred P. Sloan Foundation, the U.S. Department of Energy Office of Science, and the Participating Institutions. SDSS-IV acknowledges support and resources from the Center for High-Performance Computing at the University of Utah. The SDSS web site is www.sdss.org. SDSS-IV is managed by the Astrophysical Research Consortium for the Participating Institutions of the SDSS 
Collaboration including the Brazilian Participation Group, the Carnegie Institution for Science, Carnegie Mellon University, the Chilean Participation Group, the French Participation Group, Harvard-Smithsonian Center for Astrophysics, Instituto de Astrofísica de Canarias, The Johns Hopkins University, Kavli Institute for the Physics and Mathematics of the Universe (IPMU) / University of Tokyo, Lawrence Berkeley National Laboratory, Leibniz Institut für Astrophysik Potsdam (AIP), Max-Planck-Institut für Astronomie (MPIA Heidelberg), Max-Planck-Institut für Astrophysik (MPA Garching), Max-Planck-Institut fü Extraterrestrische Physik (MPE), National Astronomical Observatories of China, New Mexico State University, New York University, University of Notre Dame Observatário Nacional / MCTI, The Ohio State University, Pennsylvania State University, Shanghai Astronomical Observatory, UK Participation Group, Universidad Nacional Autónoma de México, University of Arizona, University of Colorado Boulder, University of Oxford, University of Portsmouth, University of Utah, University of Virginia, University of Washington, University of Wisconsin, Vanderbilt University, and Yale University.

\section{References}

Adibekyan, V. Z., Sousa, S. G., Santos, N. C., et al. 2012, A\&A, 545, A32 Adibekyan, V. Z., Benamati, L., Santos, N. C., et al. 2015, MNRAS, 450, 1900 Agertz, O., \& Kravtsov, A. V. 2015, ApJ, 804, 18

Agertz, O., Teyssier, R., \& Moore, B. 2009, MNRAS, 397, 64

Agertz, O., Teyssier, R., \& Moore, B. 2011, MNRAS, 410, 1391

Alves-Brito, A., Meléndez, J., Asplund, M., Ramírez, I., \& Yong, D. 2010, A\&A, 513, A35

Andrievsky, S. M., Martin, R. P., Kovtyukh, V. V., Korotin, S. A., \& Lépine, J. R. D. 2016, MNRAS, 461, 4256

Armillotta, L., Fraternali, F., \& Marinacci, F. 2016, MNRAS, 462, 4157

Ballero, S. K., Matteucci, F., Origlia, L., \& Rich, R. M. 2007, A\&A, 467, 123

Basu, S., Grundahl, F., Stello, D., et al. 2011, ApJ, 729, L10

Battistini, C., \& Bensby, T. 2015, A\&A, 577, A9

Battistini, C., \& Bensby, T. 2016, A\&A, 586, A49

Bell, E. F., Monachesi, A., Harmsen, B., et al. 2017, ApJ, 837, L8

Bensby, T., Feltzing, S., Lundström, I., \& Ilyin, I. 2005, A\&A, 433, 185

Bensby, T., Feltzing, S., Johnson, J. A., et al. 2010, A\&A, 512, A41

Bensby, T., Alves-Brito, A., Oey, M. S., Young, D. \& Melendez, J. 2011, ApJ, 735, L46

Bensby, T., Yee, J. C., Feltzing, S., et al. 2013, A\&A, 549, A147

Bensby, T., Feltzing, S., \& Oey, M. S. 2014, A\&A, 562, A71

Bensby, T., Feltzing, S., Gould, A., et al. 2017, A\&A, 605, A89

Bigiel, F., Leroy, A. K., Walter, F., et al. 2011, ApJ, 730, L13

Bigiel, F., Cormier, D., \& Schmidt, T. 2014, Astron. Nachr., 335, 470

Birnboim, Y., \& Dekel, A. 2003, MNRAS, 345, 349

Bland-Hawthorn, J., \& Freeman, K. C. 2004, PASA, 21, 110

Boesgaard, A. M., Lum, M. G., \& Deliyannis, C. P. 2015, ApJ, 799, 202

Bouché, N., Dekel, A., Genzel, R., et al. 2010, ApJ, 718, 1001

Bovy, J., Rix, H.-W., Liu, C., et al. 2012, ApJ, 753, 148

Bovy, J., Nidever, D. L., Rix, H.-W., et al. 2014, ApJ, 790, 127

Bovy, J., Rix, H.-W., Schlafly, E. F., et al. 2016, ApJ, 823, 30

Brogaard, K., Bruntt, H., Grundahl, F., et al. 2011, A\&A, 525, A2

Catchpole, R. M., Whitelock, P. A., Feast, M. W., et al. 2016, MNRAS, 455 2216

Carraro, G. 2014, Tenth Pacific Rim Conference on Stellar Astrophysics, 482, 245

Carraro, G., Villanova, S., Demarque, P., et al. 2006, ApJ, 643, 1151

Ceverino, D., Primack, J., Dekel, A., \& Kassin, S. A. 2017, MNRAS, 467, 2664

Cheng, J. Y., Rockosi, C. M., Morrison, H. L., et al. 2012, ApJ, 746, 149

Clarkson, W., Sahu, K., Anderson, J., et al. 2008, ApJ, 684, 1110

Cortese, L., Catinella, B., \& Janowiecki, S. 2017, ApJ, 848, L7

Daddi, E., Elbaz, D., Walter, F., et al. 2010, ApJ, 714, L118

Danovich, M., Dekel, A., Hahn, O., Ceverino, D., \& Primack, J. 2015, MNRAS, 449, 2087

Davé, R., Finlator, K., \& Oppenheimer, B. D. 2012, MNRAS, 421, 98

Davé, R., Rafieferantsoa, M. H., Thompson, R. J., \& Hopkins, P. F. 2017, MNRAS, 467, 115

Dehnen, W. 2000, AJ, 119, 800

Dekel, A., \& Birnboim, Y. 2006, MNRAS, 368, 2

Dessauges-Zavadsky, M., Zamojski, M., Schaerer, D., et al. 2015, A\&A, 577, A50

Dib, S. 2011, ApJ, 737, L20

Di Matteo, P. 2016, PASA, 33, e027

Di Matteo, P., Haywood, M., Gómez, A., et al. 2014, A\&A, 567, A122

Di Matteo, P., Gómez, A., Haywood, M., et al. 2015, A\&A, 577, A1

Dubois, Y., Pichon, C., Devriendt, J., et al. 2013, MNRAS, 428, 2885

Dutton, A. A., van den Bosch, F. C., \& Dekel, A. 2010, MNRAS, 405, 1690

Elmegreen, B. G., \& Elmegreen, D. M. 2006, ApJ, 650, 644
Förster Schreiber, N. M., Genzel, R., Lehnert, M. D., et al. 2006, ApJ, 645, 1062 Fragkoudi, F., Di Matteo, P., Haywood, M., et al. 2017, A\&A, 607, L4 Fragkoudi, F., Di Matteo, P., Haywood, M., et al. 2018, A\&A, 616, A180 Fraternali, F. 2013, IAU Symp., 298, 228

Fraternali, F., \& Tomassetti, M. 2012, MNRAS, 426, 2166

Freeman, K., \& Bland-Hawthorn, J. 2002, ARA\&A, 40, 487

Fuhrmann, K. 1998, A\&A, 338, 161

Fulbright, J. P., McWilliam, A., \& Rich, R. M. 2007, ApJ, 661, 1152

Geisler, D., Villanova, S., Carraro, G., et al. 2012, ApJ, 756, L40

Genzel, R., Tacconi, L. J., Lutz, D., et al. 2015, ApJ, 800, 20

Gonzalez, O. A., Rejkuba, M., Zoccali, M., et al. 2011, A\&A, 530, A54

Gonzalez, O. A., Zoccali, M., Vasquez, S., et al. 2015, A\&A, 584, A46

González Delgado, R. M., Pérez, E., Cid Fernandes, R., et al. 2017, A\&A, 607, A 128

Grieco, V., Matteucci, F., Pipino, A., \& Cescutti, G. 2012, A\&A, 548, A60

Groenewegen, M. A. T., \& Blommaert, J. A. D. L. 2005, A\&A, 443, 143

Hallé, A., \& Combes, F. 2013, A\&A, 559, A55

Hallé, A., Di Matteo, P., Haywood, M., \& Combes, F. 2015, A\&A, 578, A58

Hallé, A., Di Matteo, P., Haywood, M., \& Combes, F. 2018, A\&A, 616, A86

Hayden, M. R., Bovy, J., Holtzman, J. A., et al. 2015, ApJ, 808, 132

Hayward, C. C., \& Hopkins, P. F. 2017, MNRAS, 465, 1682

Haywood, M. 2001, MNRAS, 325, 1365

Haywood, M. 2008, MNRAS, 388, 1175

Haywood, M., Di Matteo, P., Lehnert, M., Katz, D., \& Gómez, A. 2013, A\&A, 560, A109

Haywood, M., Di Matteo, P., Snaith, O., \& Lehnert, M. D. 2015, A\&A, 579, A5 Haywood, M., Lehnert, M. D., Di Matteo, P., et al. 2016a, A\&A, 589, A66 Haywood, M., Di Matteo, P., Snaith, O., \& Calamida, A. 2016b, A\&A, 593, A82 Hill, V., Lecureur, A., Gómez, A., et al. 2011, A\&A, 534, A80

Hinkel, N. R., Young, P. A., Pagano, M. D., et al. 2016, ApJS, 226, 4

Ilbert, O., McCracken, H. J., Le Fèvre, O., et al. 2013, A\&A, 556, A55

Iwamoto, K., Brachwitz, F., Nomoto, K., et al. 1999, ApJS, 125, 439

Ishigaki, M. N., Aoki, W., \& Chiba, M. 2013, ApJ, 771, 67

Jílková, L., Carraro, G., Jungwiert, B., \& Minchev, I. 2012, A\&A, 541, A64

Johnson, C. I., Rich, R. M., Kobayashi, C., \& Fulbright, J. P. 2012, ApJ, 749, 175

Johnson, C. I., Rich, R. M., Kobayashi, C., et al. 2013, ApJ, 765, 157

Johnson, C. I., Rich, R. M., Kobayashi, C., Kunder, A., \& Koch, A. 2014, AJ, 148,67

Kalberla, P. M. W., \& Kerp, J. 2009, ARA\&A, 47, 27

Karakas, A. I. 2010, MNRAS, 403, 1413

Kawata, D., \& Gibson, B. K. 2003, MNRAS, 340, 908

Kereš, D., Katz, N., Weinberg, D. H., \& Davé, R. 2005, MNRAS, 363, 2

Kereš, D., Katz, N., Fardal, M., Davé, R., \& Weinberg, D. H. 2009, MNRAS, 395,160

Khoperskov, S., Haywood, M., Di Matteo, P., Lehnert, M. D., \& Combes, F 2018, A\&A, 609, A60

Kroupa, P. 2001, MNRAS, 322, 231

Krumholz, M. R., \& Dekel, A. 2012, ApJ, 753, 16

Kubryk, M., Prantzos, N., \& Athanassoula, E. 2015, A\&A, 580, A126

Kunder, A., Koch, A., Rich, R. M., et al. 2012, AJ, 143, 57

Lagos, C. D. P., Baugh, C. M., Lacey, C. G., et al. 2011, MNRAS, 418, 1649

Lecureur, A., Hill, V., Zoccali, M., et al. 2007, A\&A, 465, 799

Leitner, S. N. 2012, ApJ, 745, 149

Lehnert, M. D., Le Tiran, L., Nesvadba, N. P. H., et al. 2013, A\&A, 555, A72

Lehnert, M. D., Di Matteo, P., Haywood, M., \& Snaith, O. N. 2014, ApJ, 789, L30

Lehnert, M. D., van Driel, W., Le Tiran, L., Di Matteo, P., \& Haywood, M. 2015, A\&A, 577, A112

Leroy, A. K., Walter, F., Brinks, E., et al. 2008, AJ, 136, 2782

Leroy, A. K., Walter, F., Sandstrom, K., et al. 2013, AJ, 146, 19

Lilly, S. J., Carollo, C. M., Pipino, A., Renzini, A., \& Peng, Y. 2013, ApJ, 772, 119

Loebman, S. R., Debattista, V. P., Nidever, D. L., et al. 2016, ApJ, 818, L6

Ma, X., Hopkins, P. F., Wetzel, A. R., et al. 2017, MNRAS, 467, 2430

Majewski, S. R., Schiavon, R. P., Frinchaboy, P. M., et al. 2017, AJ, 154, 94

Maller, A. H., \& Bullock, J. S. 2004, MNRAS, 355, 694

Martig, M., Minchev, I., Ness, M., Fouesneau, M., \& Rix, H.-W. 2016, ApJ, 831, 139

McWilliam, A. 2016, PASA, 33, e040

McWilliam, A., \& Rich, R. M. 1994, ApJS, 91, 749

Meléndez, J., Asplund, M., Alves-Brito, A., et al. 2008, A\&A, 484, L21

Melvin, T., Masters, K., Lintott, C., et al. 2014, MNRAS, 438, 2882

Miller, M. J., \& Bregman, J. N. 2013, ApJ, 770, 118

Minchev, I., Chiappini, C., \& Martig, M. 2013, A\&A, 558, A9

Minchev, I., Chiappini, C., \& Martig, M. 2014, A\&A, 572, A92

Mishenina, T. V., Pignatari, M., Korotin, S. A., et al. 2013, A\&A, 552, A128

Monari, G., Famaey, B., Siebert, A., et al. 2016, MNRAS, 461, 3835 
Morishita, T., Ichikawa, T., Noguchi, M., et al. 2015, ApJ, 805, 34 Muzzin, A., Marchesini, D., Stefanon, M., et al. 2013, ApJ, 777, 18 Nataf, D. M. 2016, PASA, 33, e023

Ness, M., Freeman, K., Athanassoula, E., et al. 2013a, MNRAS, 432, 2092

Ness, M., Freeman, K., Athanassoula, E., et al. 2013b, MNRAS, 430, 836

Ness, M., Debattista, V. P., Bensby, T., et al. 2014, ApJ, 787, L19

Ness, M., Zasowski, G., Johnson, J. A., et al. 2016, ApJ, 819, 2

Nissen, P. E., \& Schuster, W. J. 2010, A\&A, 511, L10

Nomoto, K., Tominaga, N., Umeda, H., Kobayashi, C., \& Maeda, K. 2006, Nucl. Phys. A, 777, 424

Noterdaeme, P., Petitjean, P., Carithers, W. C., et al. 2012, A\&A, 547, L1

Obreschkow, D., \& Rawlings, S. 2009, MNRAS, 400, 665

Ocvirk, P., Pichon, C., \& Teyssier, R. 2008, MNRAS, 390, 1326

Pagel, B. E. J. 2009, in Nucleosynthesis and Chemical Evolution of Galaxies (Cambridge, UK: Cambridge University Press)

Papovich, C., Finkelstein, S. L., Ferguson, H. C., Lotz, J. M., \& Giavalisco, M. 2011, MNRAS, 412, 1123

Papovich, C., Labbé, I., Quadri, R., et al. 2015, ApJ, 803, 26

Papovich, C., Labbé, I., Glazebrook, K., et al. 2016, Nat. Astron., 1, 0003

Patel, S. G., van Dokkum, P. G., Franx, M., et al. 2013, ApJ, 766, 15

Peirani, S., Jung, I., Silk, J., \& Pichon, C. 2012, MNRAS, 427, 2625

Pérez-Villegas, A., Portail, M., Wegg, C., \& Gerhard, O. 2017, ApJ, 840, L2

Perret, V., Renaud, F., Epinat, B., et al. 2014, A\&A, 562, A1

Popping, G., Somerville, R. S., \& Trager, S. C. 2014, MNRAS, 442, 2398

Portail, M., Gerhard, O., Wegg, C., \& Ness, M. 2017, MNRAS, 465, 1621

Raiteri, C. M., Villata, M., \& Navarro, J. F. 1996, A\&A, 315, 105

Reddy, B. E., Tomkin, J., Lambert, D. L., \& Allende Prieto, C. 2003, MNRAS, 340, 304

Reddy, B. E., Lambert, D. L., \& Allende Prieto, C. 2006, MNRAS, 367, 1329

Robichaud, F., Williamson, D., Martel, H., Kawata, D., \& Ellison, S. L. 2017, MNRAS, 469, 3722
Rojas-Arriagada, A., Recio-Blanco, A., Hill, V., et al. 2014, A\&A, 569, A103 Ryde, N., \& Schultheis, M. 2015, A\&A, 573, A14

Ryde, N., Gustafsson, B., Edvardsson, B., et al. 2010, A\&A, 509, A20 Saintonge, A., Lutz, D., Genzel, R., et al. 2013, ApJ, 778, 2

Schinnerer, E., Groves, B., Sargent, M. T., et al. 2016, ApJ, 833, 112

Schreiber, C., Elbaz, D., Pannella, M., et al. 2016, A\&A, 589, A35

Schultheis, M., Rojas-Arriagada, A., García Pérez, A. E., et al. 2017, A\&A, 600, A14

Shen, J., Rich, R. M., Kormendy, J., et al. 2010, ApJ, 720, L72

Sheth, K., Elmegreen, D. M., Elmegreen, B. G., et al. 2008, ApJ, 675, 1141

Snaith, O. N., Haywood, M., Di Matteo, P., et al. 2014, ApJ, 781, L31

Snaith, O., Haywood, M., Di Matteo, P., et al. 2015, A\&A, 578, A87

Stinson, G. S., Dutton, A. A.,Wang, L., et al. 2015, MNRAS, 454, 1105

Tacconi, L. J., Neri, R., Genzel, R., et al. 2013, ApJ, 768, 74

Tacconi, L. J., Genzel, R., Saintonge, A., et al. 2018, ApJ, 853, 179

Tillson, H., Devriendt, J., Slyz, A., Miller, L., \& Pichon, C. 2015, MNRAS, 449, 4363

Tsujimoto, T., \& Bekki, K. 2012, ApJ, 747, 125

Uttenthaler, S., Schultheis, M., Nataf, D. M., et al. 2012, A\&A, 546, A57

Valenti, E., Zoccali, M., Renzini, A., et al. 2013, A\&A, 559, A98

Van der Swaelmen, M., Barbuy, B., Hill, V., et al. 2016, A\&A, 586, A1

van de Voort, F., Schaye, J., Booth, C. M., \& Dalla Vecchia, C. 2011, MNRAS, 415,2782

van de Voort, F., Quataert, E., Hopkins, P. F., et al. 2016, MNRAS, 463, 4533

van Dokkum, P. G., Leja, J., Nelson, E. J., et al. 2013, ApJ, 771, L35

van Loon, J. T., Gilmore, G. F., Omont, A., et al. 2003, MNRAS, 338, 857

Wang, J., Shi, J., Pan, K., et al. 2016, MNRAS, 460, 3179

Woods, R. M., Wadsley, J.,Couchman, H. M. P., Stinson, G., \& Shen, S. 2014 MNRAS, 442, 732

Zoccali, M., Vasquez, S., Gonzalez, O. A., et al. 2017, A\&A, 599, A12 


\section{Appendix A: Abundances}

Abundance ratios of elements have been used as one of the main arguments supporting the idea that the bulge and disk are two different populations. The most complete and recent review is McWilliam (2016), whose diagnosis in this regard is that the differences are real for several elements. In this appendix, we offer a different view and argue that the evidence in support of significant differences is weak.

\section{A.1. What level of systematics are expected?}

Table 1 from McWilliam (2016) shows that the abundance ratio of magnesium at $[\mathrm{Fe} / \mathrm{H}]=0$ and $[\mathrm{Fe} / \mathrm{H}]=+0.5$ has continuously decreased from (respectively) $[\mathrm{Mg} / \mathrm{Fe}] \sim+0.28$ and $[\mathrm{Mg} / \mathrm{Fe}] \sim+0.1$ dex in McWilliam \& Rich (1994) and Lecureur et al. (2007) to around +0.15 and 0.0 dex in the most recent studies. These last values are similar to what is observed on dwarfs of the inner disk observed in the solar vicinity (see e.g., Adibekyan et al. 2012, Fig. 8, red triangles). Systematic errors between different studies still exist for several elements however. The offset measured for instance by Johnson et al. (2014) between their measurements and those of Bensby et al. (2013) on silicon, calcium, sodium or nickel is about $0.1-0.2$ dex, while the agreement is good for magnesium and oxygen. In addition, while systematic and important effects on metallicities are not expected for solar vicinity samples, this is still the case for bulge stars. For instance, as mentioned above, Rojas-Arriagada et al. (2014) found that their metallicity scale is shifted by $-0.21 \mathrm{dex}$ compared to the one provided by Hill et al. (2011), which will also impact the abundance ratios. Hence, differences of the order of 0.1-0.2 dex are not unexpected also on metallicities.

Adibekyan et al. (2015) have measured the abundance ratios of different elements for a sample of giant stars and shown that these abundances can be offset by as much as $0.1 \mathrm{dex}$ compared to their sample of solar vicinity dwarfs (Adibekyan et al. 2012). This is true for $[\mathrm{Si} / \mathrm{Fe}]$, but also for $[\mathrm{Mg} / \mathrm{Fe}]$ at subsolar metallicities (with similar offsets). Fine-tuning their linelist, Adibekyan et al. (2015) were able to significantly reduce the offset between the two abundances. However, the offset they obtained should probably be considered as realistic for the differences to be expected when analyzing stars that most possibly have the same chemical patterns but are of different stellar types and/or luminosity classes. Finally, in a detailed study analyzing the spectra of four (dwarf) stars by different groups, Hinkel et al. (2016) find systematic differences in temperature and $[\mathrm{Fe} / \mathrm{H}]$ of more than $100 \mathrm{~K}$ and 0.2 dex. The differences in $[\mathrm{Fe} / \mathrm{H}]$ are still of the order of 0.1 dex when the same line list and the same atmospheric parameters are used.

Below, we examine the arguments that have been developed in the literature as they are summarized and updated by McWilliam (2016) in his review, bearing in mind that differences between studies can still be of the order of $0.1-0.2$ dex, either in abundance ratios, or in metallicities, either because of residual systematics in the analysis, or possible difference between the treatment of dwarfs versus giants.

\section{A.2. Oxygen}

McWilliam (2016, hereafter McW16) suggests that the knee in $[\mathrm{O} / \mathrm{Fe}]$ occurs at a higher metallicity in the bulge than in the disk, indicating a higher SFR and faster chemical enrichment. His analysis is based on abundances measured by Johnson et al. (2014), and locates the knee at $[\mathrm{Fe} / \mathrm{H}]=-0.25$ dex. The decrease in $[\mathrm{O} / \mathrm{Fe}]$ occurs at lower metallicities in Bensby et al. (2013; hereafter $\mathrm{B} 13 ;[\mathrm{Fe} / \mathrm{H}] \sim-0.6 \mathrm{dex}$ ), but $\mathrm{McW} 16$ seems to favor Johnson et al. because according to him alpha elements in B13 also have a knee at higher metallicities. However, we find no such evidence in Fig. 25 of B13 (see also Bensby et al. 2017), where the knee in all elements is certainly at metallicities not higher than $[\mathrm{Fe} / \mathrm{H}]=-0.5 \mathrm{dex}$ (inasmuch as something like a "knee" can really be defined, which, for $\mathrm{Si}, \mathrm{Ca}$ and even $\mathrm{Ti}$, seems vain), which means 0.25 dex lower than for oxygen in Johnson et al. (2014). This is much larger than any possible difference in the metallicity of the knee of oxygen and the other elements in B13.

\section{A.3. Alpha elements and the case of magnesium}

Figure A.1a shows the solar vicinity data for the mean of alpha elements $\mathrm{Mg}, \mathrm{Si}$ and $\mathrm{Ti}$ as a function of metallicity, together with the same quantity for microlensed dwarfs of Bensby et al. (2014), shown as colored points. On the basis of this plot, we select inner-disk stars above the line shown on the plot, with OLR and outer stars in gray. We then compare the selection of these inner disk stars to the abundances of bulge, microlensed stars from Bensby et al. (2013) for individual elements: $\mathrm{Mg}, \mathrm{Si}$, $\mathrm{Ca}$, and $\mathrm{Ti}$ and oxygen on this figure and $\mathrm{Ni}, \mathrm{Zn}, \mathrm{Cr}, \mathrm{Ba}, \mathrm{La}, \mathrm{Eu}$ on the next.

As can be seen from the alpha elements, once the inner disk stars are selected, the abundances of the disk and bulge are extremely similar. Bensby et al. (2013) mentioned that the $\mathrm{Mg}$ (and $\mathrm{Ti}$ ) abundance of the bulge may be slightly enhanced compared to his solar vicinity abundances. McW16 also advocates that for $\mathrm{Mg}$, at least, the bulge trend is measurably different from the thick disk trend based on comparison between Gonzalez et al. (2015) and Bensby et al. (2005) and Reddy et al. (2006) for the solar vicinity abundances.

As mentioned above, Table 1 of McW16 shows that, while initially found to be systematically higher than the abundances measured in the solar vicinity, the bulge abundance ratio of $[\mathrm{Mg} / \mathrm{Fe}]$ has decreased systematically over the past 10 years, as testified by the $[\mathrm{Mg} / \mathrm{Fe}]$ value at solar vicinity, measured to be +0.28 dex in McWilliam \& Rich (1994) or Lecureur et al. (2007), to $0.20 \mathrm{dex}$ in Hill et al. (2011), and $0.15 \mathrm{dex}$ in Bensby et al. (2013), Johnson et al. (2014) or Gonzalez et al. (2015). Given the enormous range of variations in the measurements of abundances in the last years, a difference of $\sim 0.05 \mathrm{dex}$ is not solid evidence of a different evolution of the bulge and thick disk, also given the fact that McW16 compares bulge abundances not with the most recent data of the solar vicinity, but with Bensby et al. (2005) and Reddy et al. (2006), which have abundance patterns that are much less well defined than in the most recent studies (e.g., Adibekyan et al. 2012; Bensby et al. 2014).

Figure A.2 compares the newest (and largest) solar vicinity abundance samples of Bensby et al. (2014) and Adibekyan et al. (2012; black dots), with inner disk stars selected as described above, and the data of Gonzalez et al. (2015) for the bulge. Two different observations can be made from these comparisons. The first one is that the bulge data of Gonzalez et al. (2015) agree well with those of Bensby et al. (2014): comparing with the newest data, there is no evidence of a systematic difference between the bulge and the solar vicinity. The second is that significant differences are still visible between the dwarf abundances of two different but state-of-the-art studies of solar vicinity stars, the magnesium abundance ratio of Adibekyan et al. (2012) being lower than Bensby et al. (2014; by $\sim 0.05 \mathrm{dex})$ at $[\mathrm{Fe} / \mathrm{H}]<-0.2 \mathrm{dex}$. Based on these plots alone, 

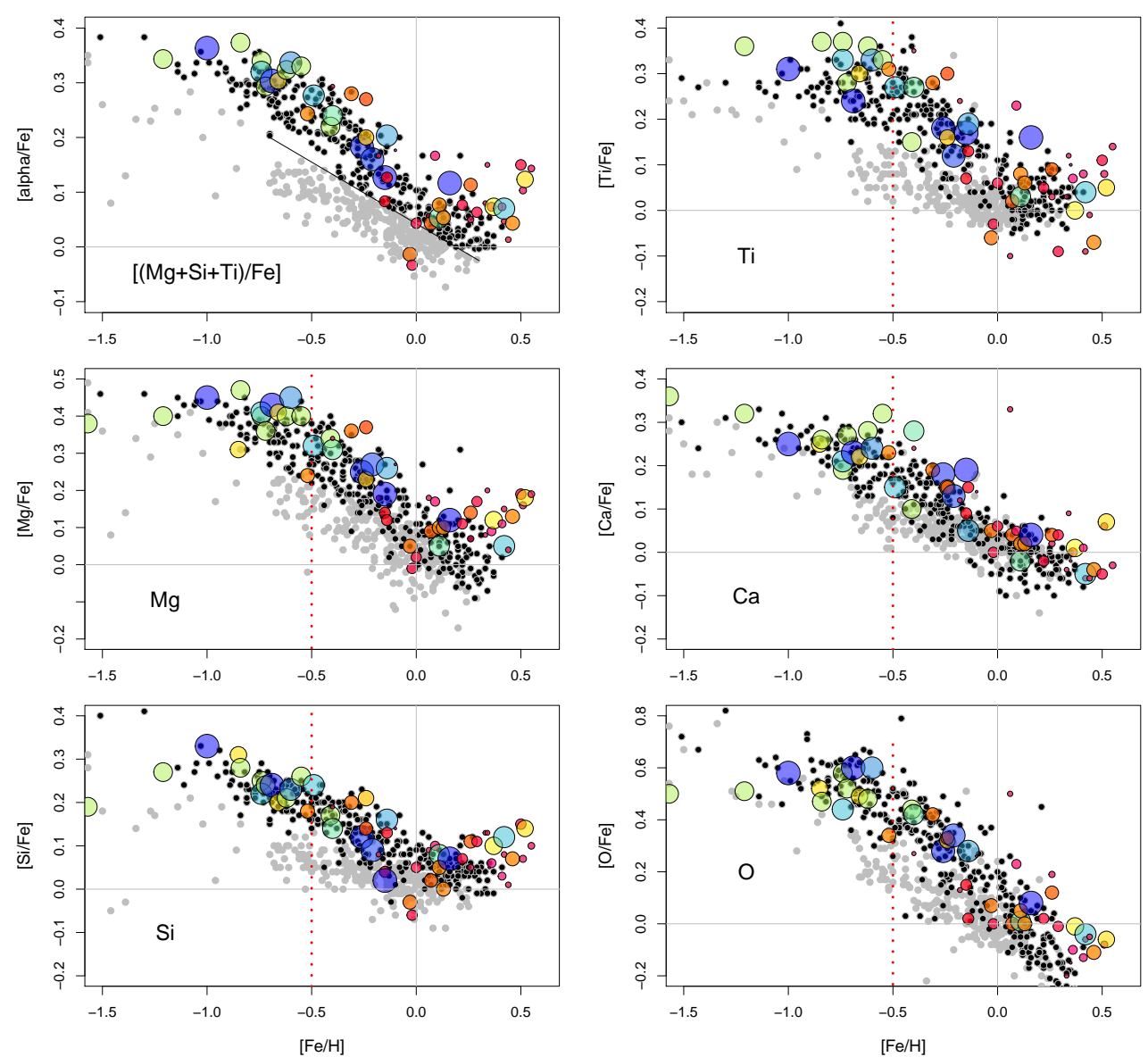

Fig. A.1. Bulge dwarfs and subgiants abundances from Bensby et al. (2013, colored circles), overlaid on stars of the solar vicinity from Bensby et al. (2014, gray and black dots) for alpha elements $(\mathrm{Mg}+\mathrm{Si}+\mathrm{Ti}), \mathrm{Al}, \mathrm{Ba}, \mathrm{Na}, \mathrm{Ni}$. The bulge data for $\mathrm{La}$ and $\mathrm{Eu}$ comes from Van der Swaelmen et al. (2016). Stars from Bensby et al. (2014) belonging to the inner disk sequence stars (and which are expected to contribute to the bulge) are selected using alpha abundances by choosing those above the black line in the plot (a). Discarding the outer disk objects (those below the line), the overlap between the inner disk stars of the solar vicinity and bulge stars is excellent. Colors and sizes of the symbols for bulge stars is coding their ages, which come from Bensby et al. (2013).

and taking for granted that differences of 0.05 dex are significant, one would suggest that the samples of Gonzalez et al. (2015) and Bensby et al. (2014) are the same population (which they may be), while Bensby et al. (2014) and Adibekyan et al. (2012) are sampling two different populations (which they are not). Hence, if state-of-the-art solar vicinity samples (of dwarfs) still show offsets of about 0.05 dex, why should we take similar differences between bulge and solar vicinity stars (made, in the case of Johnson et al. 2014, of giants) as significant?

An important reason why McW16 finds the magnesium abundance of the bulge to be enhanced compared to the solar vicinity is that he compares with the sample of Bensby et al. (2005), which lacks a clear thick disk sequence. Hence, while McW16 finds that $[\mathrm{Mg} / \mathrm{Fe}]$ at solar metallicity is 0.09 dex higher in the bulge compared to the solar vicinity stars, we see no significant differences in Fig. A.2.

Some elements have notably suffered systematic offsets, but the situation is improving. For instance, $\mathrm{Al}$ is measured in Fulbright et al. (2007) to be systematically higher in the bulge than in the disk by $\sim 0.2$ dex. In Johnson et al. (2014) or Bensby et al. (2013), it is well compatible with the disk data.

\section{A.4. Iron peak elements, $\mathrm{Zn}, \mathrm{Ni}$}

Figure A.3 (left colmun) compares zinc, nickel and chrome from B13 and B14, with the same selection of "inner disk" stars (black dots).
$\mathrm{Cu}$ is measured only by Johnson et al. (2012) in the bulge, which Johnson find to differ from solar vicinity data. Other element trends of Johnson et al. (2012) also differ from other bulge data. This is the case for instance for $[\mathrm{Na} / \mathrm{Fe}]$, which is measured to be the lowest at $[\mathrm{Fe} / \mathrm{H}]$ between -0.8 and -1.0 dex in Johnson et al. (2012; at $-0.6<[\mathrm{Na} / \mathrm{Fe}]<-0.3 \mathrm{dex})$, while it is maximum in Bensby et al. (2013) with $[\mathrm{Na} / \mathrm{Fe}] \sim+0.1$ dex at the same metallicities. Given the various ways in which bulge abundances have been modified in recent years, as illustrated in this section, it is safer to await for new measurements either to confirm the results of Johnson et al. (2012).

\section{A.5. Neutron capture elements, La, Eu, Ba}

Figure A.3 shows the comparison for Ba, also available from Bensby et al. (2013), and Eu and La from Van der Swaelmen et al. (2016) for the bulge, with measurements of the Bensby et al. (2014) sample coming from Battistini \& Bensby (2016). Contrarily to Van der Swaelmen etal. (2016), who compare to Bensby et al. (2005) and Reddy et al. (2006), we do not see that La or Ba are enhanced compared to thick disk stars, nor that they are underabundant at higher metallicities.

Van der Swaelmen et al. (2016) suggest that s-process elements are different for bulge and disk stars, the former showing a decreasing trend with increasing metallicity for $\mathrm{Ba}, \mathrm{La}, \mathrm{Ce}$ and $\mathrm{Nd}$, being above zero at metallicities below solar, and below zerofor metallicities above solar. The study of Battistini \& Bensby (2016; 

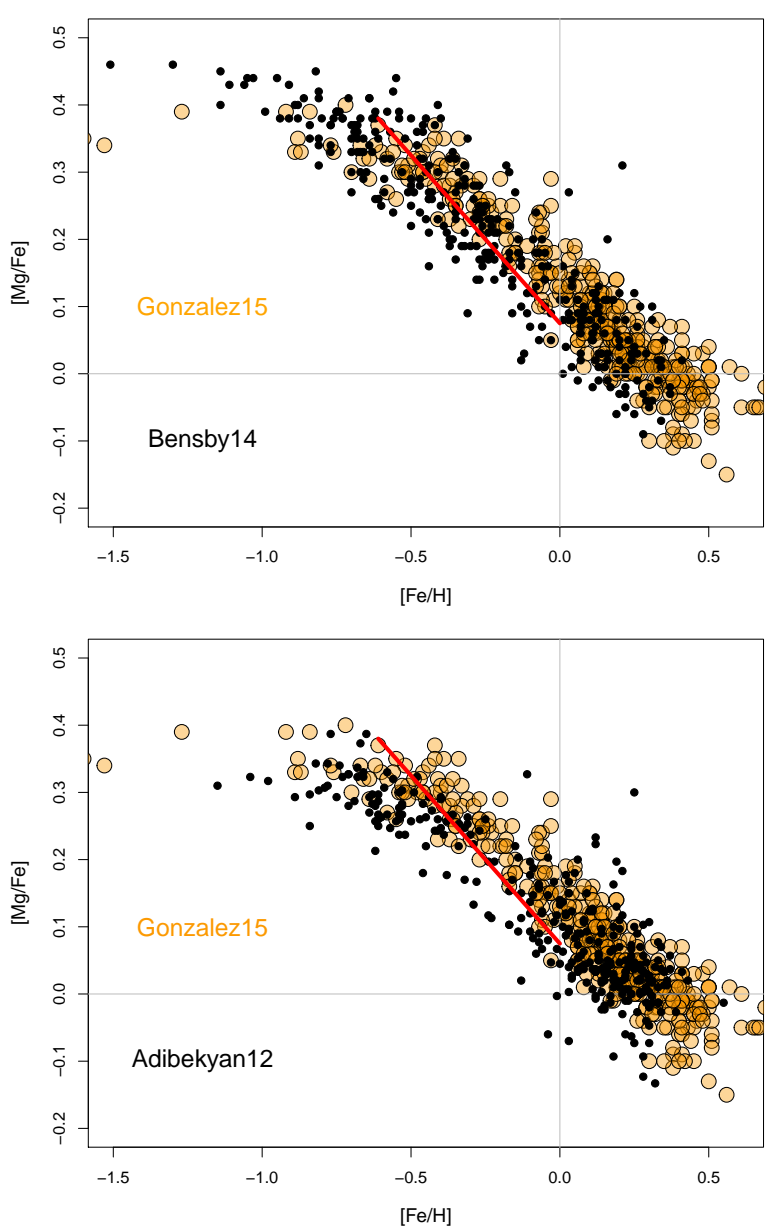

Fig. A.2. Data from Gonzalez et al. (2015; orange circles) together with "inner disk" stars of the solar vicinity from Bensby et al. (2014) and Adibekyan et al. (2012), where "inner disk" stars have been selected in the same way. We note the difference in $[\alpha / \mathrm{Fe}]$ element sequence between Bensby et al. (2014) and Adibekyan et al. (2012). See text for comments. not available to Van der Swaelmen et al. 2016) shows that these trends are closely followed by solar vicinity stars; see their Fig. 2, for $\mathrm{La}, \mathrm{Ce}, \mathrm{Nd}$. At high metallicities, Van der Swaelmen et al. (2016) show lower $[\mathrm{Ba} / \mathrm{Fe}]$ abundances than B14. However, the $[\mathrm{Ba} / \mathrm{Fe}]$ bulge data of B13 are in perfect agreement with B14 (see Fig. A.3), casting some doubts on the idea that the differences between Van der Swaelmen et al. (2016) and B14 are real.

(a) $[\mathrm{La} / \mathrm{Eu}]$. Johnson et al. (2012) argued that the ratio of $[\mathrm{La} / \mathrm{Eu}]$ is lower in the bulge than in the disk and that it indicates that the majority of the bulge formed rapidly $(<1 \mathrm{Gyr})$. This is also claimed by McW16, comparing the bulge data from Johnson et al. (2012), MFR10 sample, and Van der Swaelmen et al. (2016) to the more recent solar vicinity measurements of Battistini \& Bensby (2016).

However, we would argue first that the difference between the two bulge samples of Johnson et al. (2012) and Van der Swaelmen et al. (2016) seem (pink and blue symbols respectively) no less important that seen between solar vicinity stars by McW16 and Johnson et al. (2012; black and pink symbols, respectively), and for the same reason: Johnson et al. (2012) is clearly undersampling metal-rich stars, which are much better represented in Van der Swaelmen et al. (2016) or in solar vicinity samples. This is illustrated in our Fig. A.4, which compares the $[\mathrm{La} / \mathrm{Eu}]$ abundance ratio for the two samples from Johnson et al. (2012) and Van der Swaelmen et al. (2016), with three different samples of solar vicinity stars from Battistini \& Bensby (2015), Mishenina et al. (2013) and Ishigaki et al. (2013). There is good overlap between the bulge (colored symbols) and solar vicinity (black symbols) samples, and the two populations are perfectly compatible. We conclude that the available $[\mathrm{La} / \mathrm{Eu}]$ abundance ratios do not provide evidence that the bulge formed in a manner different from the inner disk. 
M. Haywood et al.: Phylogeny of the Milky Way's inner disk and bulge populations
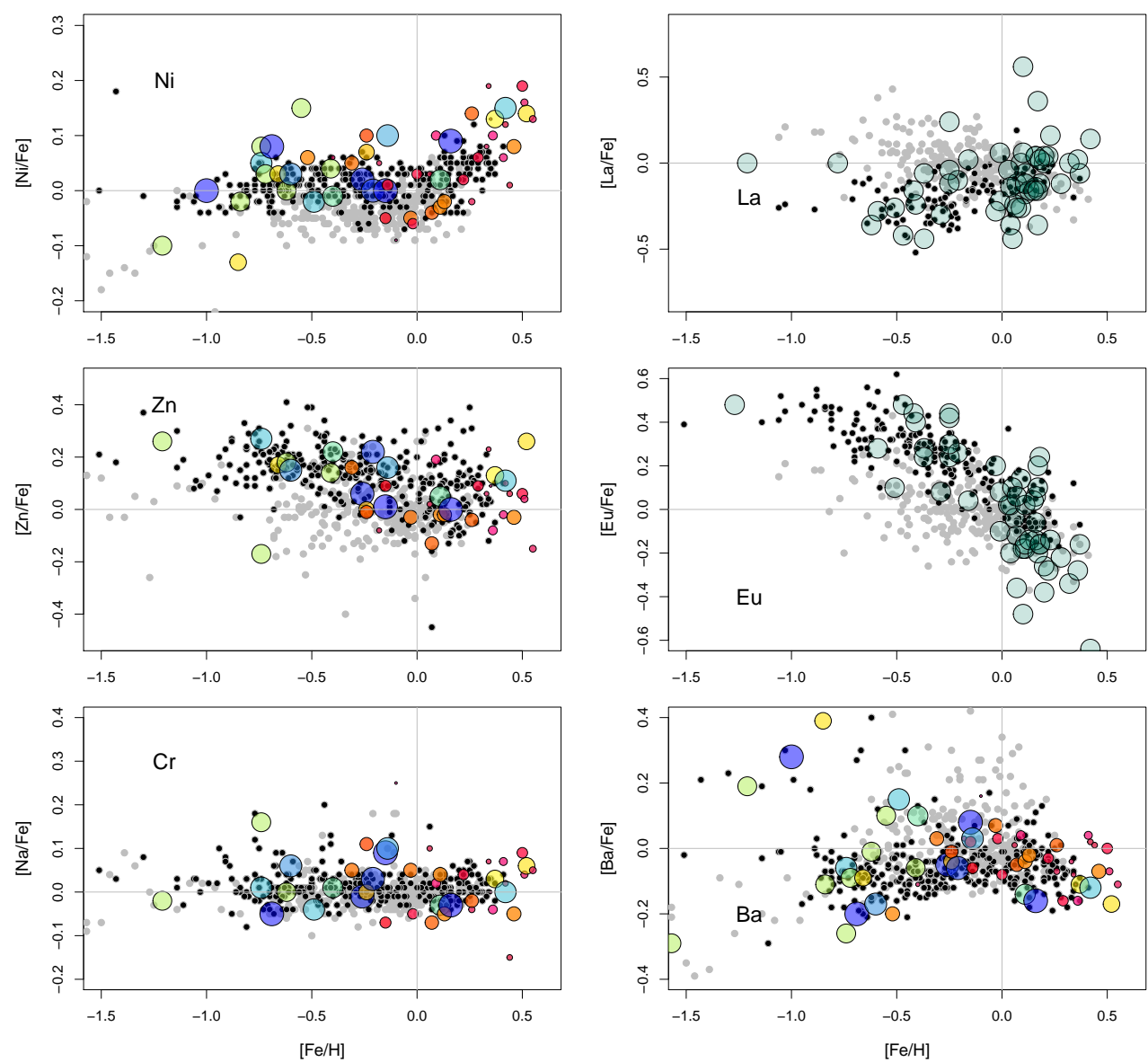

Fig. A.3. As in Fig. A.1 for iron-peak elements (left column) $\mathrm{Ni}, \mathrm{Zn}$ and $\mathrm{Cr}$, and neutron-capture elements (right column) $\mathrm{La}, \mathrm{Eu}, \mathrm{Ba}$. The bulge data for $\mathrm{Ni}, \mathrm{Zn}, \mathrm{Cr}$ and Ba come from Bensby et al. (2013), $\mathrm{La}$ and $\mathrm{Eu}$ from Van der Swaelmen et al. (2016), while solar vicinity data for La, Eu come from Battistini \& Bensby (2016) and Ba from Bensby et al. (2014).

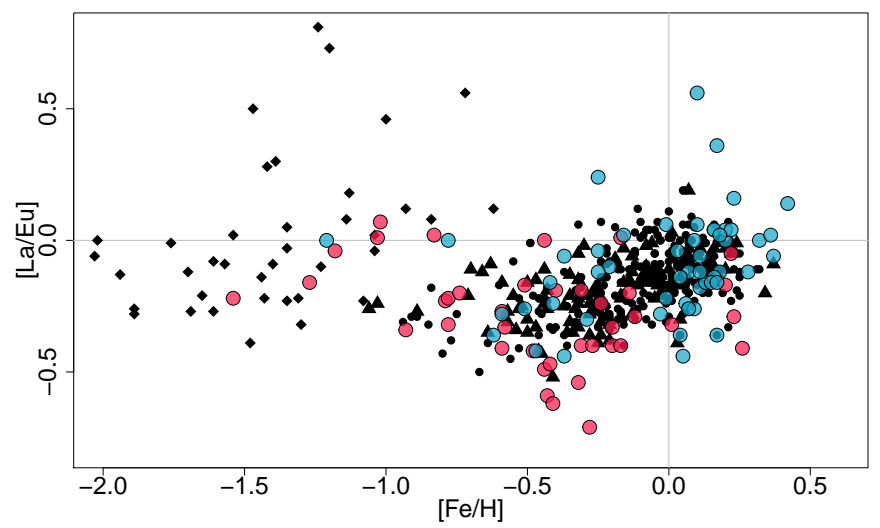

Fig. A.4. [La/Eu] from Johnson et al. (2012) and Van der Swaelmen et al. (2016; respectively pink and blue larger symbols) of bulge stars compared to data from Battistini \& Bensby (2015; triangles), Mishenina et al. (2013; squares) and Ishigaki et al. (2013; diamonds) for solar vicinity stars. There is no evidence from these data that the bulge (colored symbols) and the solar vicinity (black symbols) distributions are different. 